\title{
High scale boundary conditions in models with two Higgs doublets
}

\author{
John McDowall" and David J. Miller \\ SUPA, School of Physics and Astronomy, University of Glasgow, Glasgow G12 8QQ, United Kingdom
}

(Received 26 October 2018; published 15 July 2019)

\begin{abstract}
We investigate high scale boundary conditions on the quartic Higgs couplings and their $\beta$ functions in the type-II two Higgs doublet model and the inert doublet model. These conditions are associated with two possible UV physics scenarios: the multiple point principle, in which the potential exhibits a second minimum at $M_{\mathrm{Pl}}$, and asymptotic safety, where the scalar couplings run toward an interacting UV fixed point at high scales. We employ a renormalization group running at two loops and apply theoretical and experimental constraints to their parameter spaces. We find neither model can simultaneously accommodate the multiple point principle while also providing realistic masses for both the Higgs and the top quark. However, we do find regions of parameter space compatible with asymptotic safety.
\end{abstract}

DOI: 10.1103/PhysRevD.100.015018

\section{INTRODUCTION}

The discovery of the Higgs at ATLAS [1] and CMS [2] supports the Standard Model's (SM) mechanism of breaking the $S U(2) \times U(1)$ electroweak symmetry, which requires a single $S U(2)$ complex scalar doublet and results in one neutral scalar particle. The simplicity of the SM scalar sector is striking given the complexity of its fermion sector, so it is no surprise that the notion of extending the SM with additional scalar fields has motivated much of modern particle physics research.

In a recent work we looked at the possibility of high scale boundary conditions arising in the complex singlet extension of the SM [3]. This was motivated in part by the very small value of both the SM Higgs quartic coupling $\lambda$ and its $\beta$ function $\beta_{\lambda}$ at the Planck scale $M_{\mathrm{Pl}}$. The possibility that this interesting feature of the SM is a high scale boundary condition derived from additional physics at $M_{\mathrm{Pl}}$, and its consequences for, e.g., vacuum stability, have been extensively investigated [4-15].

Another simple way to extend the SM is to add a second Higgs doublet. Supersymmetry is a common motivation for this addition, but supersymmetric models often require finetuning of parameters or considerable complications in order to predict a Higgs mass compatible with the combined ATLAS and CMS value of $m_{h}=125.09 \pm 0.23 \mathrm{GeV}$ [16-18]. In general, the two Higgs doublet model (2HDM) must account for the very SM-like nature of the Higgs [16,19-21] while evading strong experimental bounds on its interactions.

\footnotetext{
j.mcdowall.1@ research.gla.ac.uk

david.j.miller@glasgow.ac.uk
}

Published by the American Physical Society under the terms of the Creative Commons Attribution 4.0 International license. Further distribution of this work must maintain attribution to the author(s) and the published article's title, journal citation, and DOI. Funded by SCOAP.
The aim of this work is to consider whether the inclusion of an extra Higgs doublet is compatible with both the existence of particular boundary conditions at the Planck scale and current theoretical and experimental constraints. In Sec. II we will describe our two considered models, the type-II 2HDM and the inert doublet model (IDM). In Sec. III we will then describe our methodology, including the theoretical and experimental constraints we apply to our scenarios. We will present our results for both models when confronted with boundary conditions for each of the multiple point principle (MPP) or asymptotic safety in Sec. IV. We will find that neither model can accommodate the high scale boundary conditions of the MPP, while asymptotic safety remains viable. We will draw our conclusions in Sec. V. Finally, in Appendix, we will include the renormalization group equations (RGEs) of the Higgs quartic couplings for the reader's convenience.

\section{CONSIDERED MODELS}

In this study we will focus on the type-II $2 \mathrm{HDM}$ and the IDM, and present a brief summary of the models here in order to fix our notations and conventions. For useful reviews of these models see Refs. [22] and [23], respectively.

\section{A. The two Higgs doublet model}

The most general potential of the $2 \mathrm{HDM}$ is

$$
\begin{aligned}
V\left(H_{1}, H_{2}\right)= & m_{11}^{2} H_{1}^{\dagger} H_{1}+m_{22}^{2} H_{2}^{\dagger} H_{2}-\left(m_{12}^{2} H_{1}^{\dagger} H_{2}+\text { c.c. }\right) \\
& +\lambda_{1}\left(H_{1}^{\dagger} H_{1}\right)^{2}+\lambda_{2}\left(H_{2}^{\dagger} H_{2}\right)^{2} \\
& +\lambda_{3}\left(H_{1}^{\dagger} H_{1}\right)\left(H_{2}^{\dagger} H_{2}\right)+\lambda_{4}\left(H_{1}^{\dagger} H_{2}\right)\left(H_{2}^{\dagger} H_{1}\right) \\
& +\left(\frac{\lambda_{5}}{2}\left(H_{1}^{\dagger} H_{2}\right)^{2}+\lambda_{6}\left(H_{1}^{\dagger} H_{1}\right)\left(H_{1}^{\dagger} H_{2}\right)\right. \\
& \left.+\lambda_{7}\left(H_{2}^{\dagger} H_{2}\right)\left(H_{1}^{\dagger} H_{2}\right)+\text { c.c. }\right),
\end{aligned}
$$


where the two Higgs doublets themselves are given by

$$
H_{n}=\left(\begin{array}{c}
\chi_{n}^{+} \\
\left(H_{n}^{0}+i A_{n}^{0}\right) / \sqrt{2}
\end{array}\right), \quad n=1,2 .
$$

The parameters $m_{11}^{2}, m_{22}^{2}$, and $\lambda_{1,2,3,4}$ are real, while $m_{12}^{2}$ and $\lambda_{5,6,7}$ can in principle be complex and induce $C P$ violation. During electroweak symmetry breaking the neutral components of the Higgs fields, $H_{n}^{0}$, develop vacuum expectation values (VEVs) $\left\langle H_{n}^{0}\right\rangle=v_{n} / \sqrt{2}$. The expression $v=\sqrt{v_{1}^{2}+v_{2}^{2}}$ is set to the SM Higgs VEV's value of $246 \mathrm{GeV}$, but the ratio of the VEVs, $\tan \beta=v_{2} / v_{1}$, is a free parameter. The physical scalar sector of the model includes two neutral scalar Higgs $h$ and $H$, a pseudoscalar Higgs $A$, and the charged Higgs $H^{ \pm}$.

It is clear that the $2 \mathrm{HDM}$ potential is considerably more complicated than its Standard Model counterpart, so it is common to employ additional global symmetries to increase the predictivity of the model. There are only six possible types of global symmetry that have a distinctive effect on the potential [24,25]. In this work we implement a $\mathbb{Z}_{2}$ symmetry to forbid flavor changing neutral currents (FCNCs) by allowing only one type of fermion to couple to one Higgs doublet. This requirement sets $\lambda_{6}, \lambda_{7}$, and $m_{12}^{2}$ to zero. However, we then softly break this $\mathbb{Z}_{2}$ by reintroducing a positive nonzero $m_{12}^{2}$. For the results reported here we will restrict ourselves to a type II model where up-type quarks and leptons couple to the first Higgs doublet and down-type quarks to the second Higgs doublet. The dominant effect of the Yukawa sector on the running of the relevant Higgs parameters arises from the top-quark coupling, so we expect our results to be similar for other 2HDM types. We checked this by repeating the analysis for the type-I and flipped 2HDMs and found no significant differences from the results presented here.

For each parameter point the model is described by the parameters $m_{11}^{2}$ and $m_{22}^{2}$, which are replaced by $M_{Z}$ and $\tan \beta$ by applying the electroweak vacuum minimization conditions, as well as the additional input parameters, $m_{12}^{2}$ and $\lambda_{i}\left(M_{\mathrm{Pl}}\right)$ with $i=1, \ldots, 5$. We also use the top pole mass $m_{t}$ and the strong coupling constant $\alpha_{S}\left(M_{Z}\right)$ as inputs, allowing them to vary between $\pm 3 \sigma$ of their central values to account for the effect of their uncertainty on our results. Since we are interested in both the high and the low scale behavior of the potential's parameters we use SARAH 4.12.2 [26] to calculate the two-loop $\beta$ functions, which are used by FLEXIBLESUSY 2.0.1 [27-30] to run the couplings between $M_{Z}$ and $M_{\mathrm{Pl}}$.

\section{B. The inert doublet model}

We also consider the model where we introduce an additional unbroken $\mathbb{Z}_{2}$ symmetry, under which the new Higgs doublet has odd parity but all other fields are even. The scalar sector now consists of the SM Higgs field $H$ and an inert doublet $\Phi$, with mixing between the two forbidden by the new symmetry. The inert doublet does not couple to any of the SM fields and does not gain a vacuum expectation value. The potential is

$$
\begin{aligned}
V(H, \Phi)= & m_{11}^{2} H^{\dagger} H+m_{22}^{2} \Phi^{\dagger} \Phi+\lambda_{1}\left(H^{\dagger} H\right)^{2}+\lambda_{2}\left(\Phi^{\dagger} \Phi\right)^{2} \\
& +\lambda_{3}\left(H^{\dagger} H\right)\left(\Phi^{\dagger} \Phi\right)+\lambda_{4}\left(H^{\dagger} \Phi\right)\left(\Phi^{\dagger} H\right) \\
& +\left(\frac{\lambda_{5}}{2}\left(H^{\dagger} \Phi\right)^{2}+\text { H.c. }\right)
\end{aligned}
$$

where all the parameters are real. Note that now the mixing term proportional to $m_{12}^{2}$ is absent. During electroweak symmetry breaking the neutral component of the SM Higgs doublet acquires a vacuum expectation value $v \approx 246 \mathrm{GeV}$. The neutral Higgs $h$ corresponds to the SM Higgs boson while $H, A$, and $H^{ \pm}$are inert scalars. The lightest of these $h_{\text {LOP }}$ (lightest odd particle) is stable thanks to the $\mathbb{Z}_{2}$ symmetry and, assuming $h_{\mathrm{LOP}}$ is one of the neutral scalars $H$ or $A$, it is a potential Dark Matter (DM) candidate $[31,32]$.

The tree-level masses for the scalars are given by [33]

$$
\begin{aligned}
m_{h}^{2} & =m_{11}^{2}+3 \lambda_{1} v^{2}, \\
m_{H}^{2} & =m_{22}^{2}+\frac{1}{2}\left(\lambda_{3}+\lambda_{4}+\lambda_{5}\right) v^{2}, \\
m_{A}^{2} & =m_{22}^{2}+\frac{1}{2}\left(\lambda_{3}+\lambda_{4}-\lambda_{5}\right) v^{2}, \\
m_{H^{ \pm}}^{2} & =m_{22}^{2}+\frac{1}{2} \lambda_{3} v^{2} .
\end{aligned}
$$

As in the previous case, we fix the mass term associated with the SM Higgs doublet $m_{11}^{2}$ via the electroweak minimization conditions, but now do not have a second VEV to fix $m_{22}^{2}$, which must remain an input. Our input parameters are therefore $m_{22}^{2}$ and $\lambda_{i}\left(M_{\mathrm{Pl}}\right)$ with $i=1, \ldots, 5$. As in the type-II model, we use SARAH and FLEXIBLESUSY to calculate the mass spectrum and to run couplings between the low and high scales of interest.

\section{NUMERICAL ANALYSIS AND CONSTRAINTS}

The main focus of this work is the possibility and consequences of boundary conditions on all or some of the quartic couplings of the 2HDM and the IDM and their $\beta$ functions at the Planck scale,

$$
\lambda_{i}\left(M_{\mathrm{Pl}}\right), \beta_{\lambda_{i}}\left(M_{\mathrm{Pl}}\right)=0, \quad i=1, \ldots, 5 .
$$

We use SARAH 4.12.2 [26] to calculate all of the model parameters, including mass matrices, tadpole equations, vertices and loop corrections, as well as the two-loop $\beta$ functions for each model. FleXIBLESUSY 2.0.1 [27-30] uses this output to calculate the mass spectrum and to 
TABLE I. Input parameter ranges for the numerical analysis of the (left) type-II 2HDM and (right) IDM. Note that in the above, $m_{12}$ and $m_{22}$ are understood to be the square roots of the input parameters $m_{12}^{2}$ and $m_{22}^{2}$, respectively.

\begin{tabular}{lc}
\hline \multicolumn{2}{c}{ Type-II model input } \\
\hline$\lambda_{1,2}\left(M_{\mathrm{Pl}}\right)$ & $0.0-1.0$ \\
$\lambda_{3,4}\left(M_{\mathrm{Pl}}\right)$ & $-1.0-1.0$ \\
$\lambda_{5,6,7}\left(M_{\mathrm{Pl}}\right)$ & 0.0 \\
$m_{12}$ & $0.0-2000 \mathrm{GeV}$ \\
$\tan \beta$ & $2.0-50$ \\
\hline \multicolumn{2}{c}{ Inert model input } \\
\hline$\lambda_{1,2}\left(M_{\mathrm{Pl}}\right)$ & $0.0-1.0$ \\
$\lambda_{3,4}\left(M_{\mathrm{Pl}}\right)$ & $-1.0-1.0$ \\
$\lambda_{5}\left(M_{\mathrm{Pl}}\right)$ & 0.0 \\
$m_{22}$ & $0.0-2000 \mathrm{GeV}$ \\
\hline \hline
\end{tabular}

run the couplings between $M_{Z}$ and the Planck scale. Table I shows the input parameter ranges used in our scans for both the type-II and the inert models.

Valid points in our parameter space scan are required to be perturbative up to the Planck scale. For the Higgs quartic couplings this requires them to satisfy $\lambda_{i}<\sqrt{4 \pi}$ up to $M_{\mathrm{Pl}}$. We require the potential to be bounded from below at all scales up to $M_{\mathrm{Pl}}$ [34]. To that end we check if the conditions [22],

$$
\begin{aligned}
\lambda_{1} & >0, \\
\lambda_{2} & >0, \\
\lambda_{3} & >-2 \sqrt{\lambda_{1} \lambda_{2}}, \\
\lambda_{3}+\lambda_{4}-\left|\lambda_{5}\right| & >-2 \sqrt{\lambda_{1} \lambda_{2}},
\end{aligned}
$$

are met at all scales [35,36]. We use Vevacious [37] to check if the electroweak symmetry breaking minimum is the global minimum. Additionally, we require valid points to provide a SM Higgs candidate $124.7 \leq m_{h} \leq 127.1 \mathrm{GeV}$, where the allowed mass range is larger than the experimental error to additionally account for theoretical uncertainties.

Our aim is to find parameter choices that are compatible with perturbativity, vacuum stability, and the SM Higgs mass, as well as other constraints on the Higgs boson from LHC Run-I, LEP, and the Tevatron. We use 2HDMC 1.7.0 [38] to calculate the relevant branching ratios required by HigGsBounds 4.3.1 [39] to apply 95\% confidence exclusions. This same input is also used by HigGSSignals 1.4.0 [40] to perform a $\chi^{2}$ fit to the observed SM signal at the LHC. $^{1}$

In the case of the IDM we also apply constraints from analyses of LEP data [33]. Potential invisible decays of the

\footnotetext{
${ }^{1}$ We note that new beta versions of HigGsBounds-5 and HiggsSignALs-2 that include $13 \mathrm{TeV}$ LHC data were made available after this analysis was completed.
}

$W$ and $Z$ bosons via $W^{ \pm} \rightarrow A H^{ \pm}, W^{ \pm} \rightarrow H H^{ \pm}, Z \rightarrow A H$, and $Z \rightarrow H^{+} H^{-}$are ruled out by the precise measurement of the $W$ and $Z$ boson widths. To prevent these, we require $[41,42]$

$$
\begin{aligned}
& \operatorname{Min}\left(M_{A}, M_{H}\right)+M_{H^{ \pm}}>M_{W}, \\
& M_{A}+M_{H}>M_{Z}, \quad \text { and } 2 M_{H^{ \pm}}>M_{Z} .
\end{aligned}
$$

LEP constraints from searches for charginos and neutralinos $[43,44]$ are applied by excluding the region where $M_{A}<100 \mathrm{GeV}, M_{H}<80 \mathrm{GeV}$, and $M_{A}-M_{H}>8 \mathrm{GeV}$ simultaneously. To ensure that our lightest odd particle is a neutral DM candidate we also insist on the following relation between the dark sector particles:

$$
M_{H^{ \pm}}>\min \left(M_{H}, M_{A}\right) .
$$

We also look at constraints from electroweak precision observables for both of our models. The $S, T$, and $U$ parameters are calculated using $2 \mathrm{HDMC}$, and the results are checked against the current PDG limits [45], where we require these precision observables within the range of $\pm 3 \sigma$. However, we note that these constraints do not restrict the parameter space beyond the bounds arising from the LHC Run-I, LEP, and Tevatron described above.

In the 2HDM the existence of the charged Higgs bosons $H^{ \pm}$can affect the calculation of flavor observables. To take this into account we use SuPERIso [46-48] to calculate the radiative $B$ meson decay $B \rightarrow X_{s} \gamma$, the leptonic $B$ decays $B_{s}^{0} \rightarrow \mu^{+} \mu^{-}, B_{d}^{0} \rightarrow \mu^{+} \mu^{-}$, and $B \rightarrow \tau \nu$, the leptonic $D$ decays $D \rightarrow \mu \nu, D_{s} \rightarrow \mu \nu$, and $D_{s} \rightarrow \tau \nu$ as well as the semileptonic decay $B \rightarrow D \tau \nu$, the kaon decay $K \rightarrow \mu \nu$ and the pion decay $\pi \rightarrow \mu \nu$. We then apply $95 \%$ confidence level constraints on the branching ratios of these decays.

For the IDM, we use MICROMEGAS [49] to calculate the DM relic density $\Omega h^{2}$, using the lightest of the neutral scalars $H$ and $A$ as the stable DM candidate. We compare the result to the combined experimental result from the WMAP [50] and Planck [51] experiments,

$$
\Omega h^{2}=0.1199 \pm 0.0027
$$

We pass points that give a value less than $\Omega h^{2}+3 \sigma$ to allow for the possibility that the scalar DM candidate is not the only contribution to the relic density.

DM direct detection experiments place constraints on the spin independent scattering cross section of weakly interacting massive particles (WIMPs) on nucleons. The strongest of these comes from the LUX [52] and XENON1T [53] experiments, which give constraints that are dependent on the mass of the WIMP DM candidate. We use MICROMEGAS to calculate the scattering cross sections for each of the points in our scan and exclude those that give values greater than the XENON1T constraints. 


\section{RESULTS}

\section{A. The multiple point principle in the type-II two Higgs doublet model}

There are a number of possible scenarios that may enforce particular boundary conditions on the quartic Higgs couplings and their $\beta$ functions at the Planck scale [54]. One such scenario is the MPP [55] which posits that the effective potential has an additional minimum at the Planck scale, degenerate to the electroweak minimum. Applying the MPP in the SM leads to a prediction of the Higgs mass of $m_{h}=129 \pm 1.5 \mathrm{GeV}$ [17], which is not compatible with our current experimental value of $m_{h}$ but is close enough to have inspired a number of investigations into the MPP in extensions of the SM [56-59] and the 2HDM [60-62]. The simplest scenario implementation of the MPP would be to have a global minimum at a high scale $\Lambda$, degenerate with the electroweak minimum, where all of the quartic couplings are zero at $\Lambda$, e.g., $\lambda_{i}=0$, $i=1, \ldots, 5$. However, the RGE running of $\lambda_{1}$ and $\lambda_{2}$ results in an unstable vacuum configuration [60-62].

It is possible for degenerate vacua to exist within the $2 \mathrm{HDM}$ if we relax the condition $\lambda_{i}=0$. Specifically, by allowing $\lambda_{1}, \lambda_{2}, \lambda_{3}$, and $\lambda_{4}$ to be nonzero at $\Lambda$, the following conditions [60] are consistent with the implementation of the MPP at $\Lambda$ :

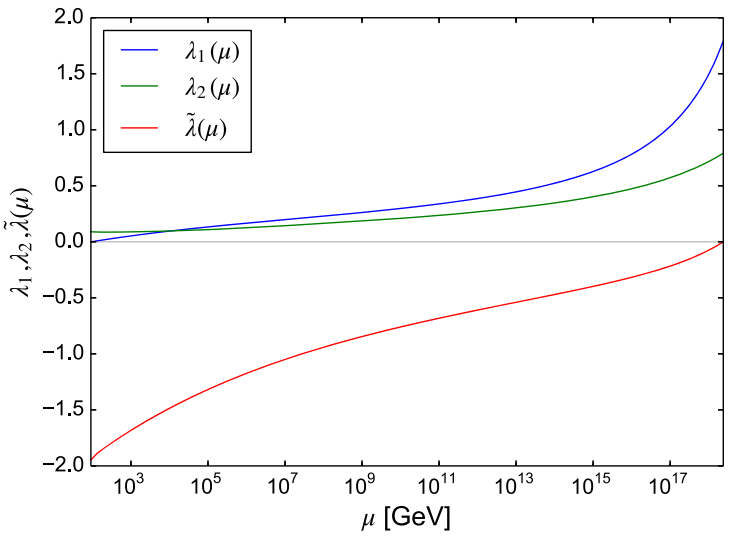

(a)

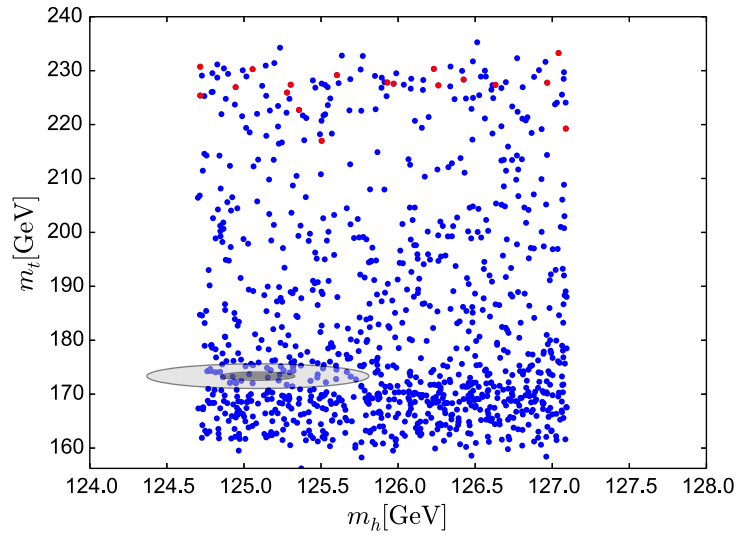

(b)

FIG. 1. (a) Example running of $\lambda_{1}, \lambda_{2}$, and $\tilde{\lambda}$ for a point that provides valid masses for the SM Higgs and the top quark in the type-II 2HDM. Boundedness from below and vacuum stability require that all three couplings are positive at all scales. (b) Results of our MPP scan in the $m_{h}-m_{t}$ plane of the type-II 2HDM. The blue points provide valid SM Higgs masses while the red points also pass the vacuum stability conditions at all scales. The ellipses show the experimentally allowed values of $m_{t}$ and $m_{h}$ at $1 \sigma$ (dark grey) and $3 \sigma$ (light grey) uncertainty.

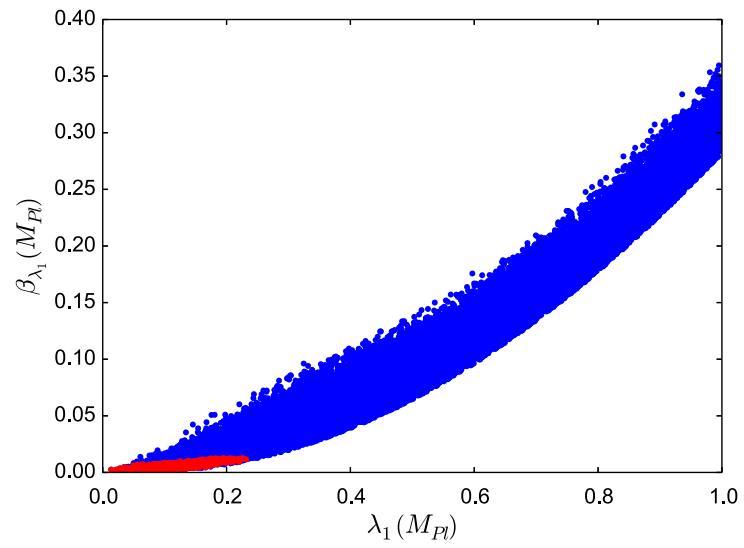

(a)

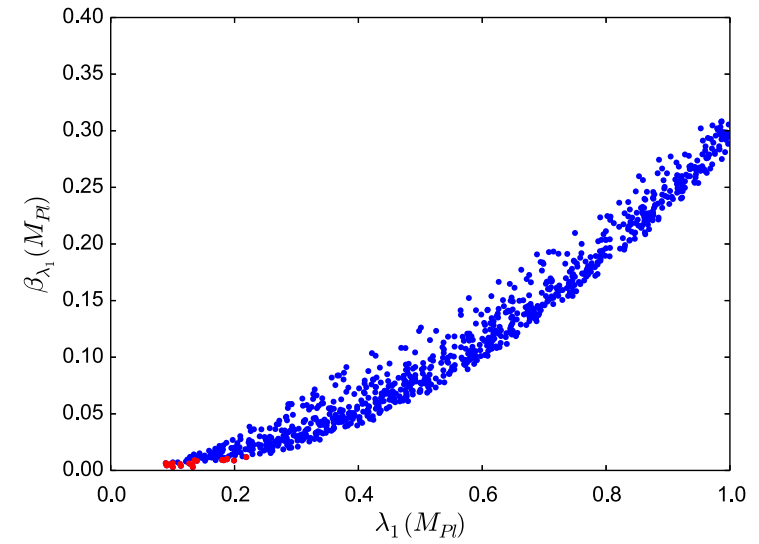

(b)

FIG. 2. Compatible values of the Higgs quartic coupling $\lambda_{1}\left(M_{\mathrm{Pl}}\right)$ against $\beta_{\lambda_{1}}\left(M_{\mathrm{Pl}}\right)$ in the type II $2 \mathrm{HDM}$. (a) Includes points that are stable and perturbative up to $M_{\mathrm{Pl}}$ and includes an SM Higgs candidate, while (b) also enforces all relevant experimental constraints discussed in Sec. III. Blue points obey $\beta_{\lambda_{1,2,3,4}}<1.0$ at $M_{\mathrm{Pl}}$ while red points obey $\beta_{\lambda_{1}}<0.0127, \beta_{\lambda_{2}}<0.0064, \beta_{\lambda_{3}}<0.0139, \beta_{\lambda_{4}}<$ 0.0030 at $M_{\mathrm{Pl}}$. 


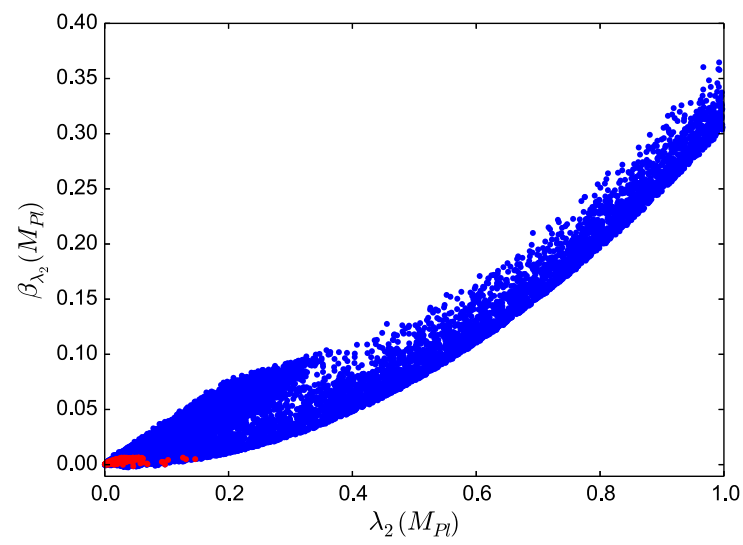

(a)

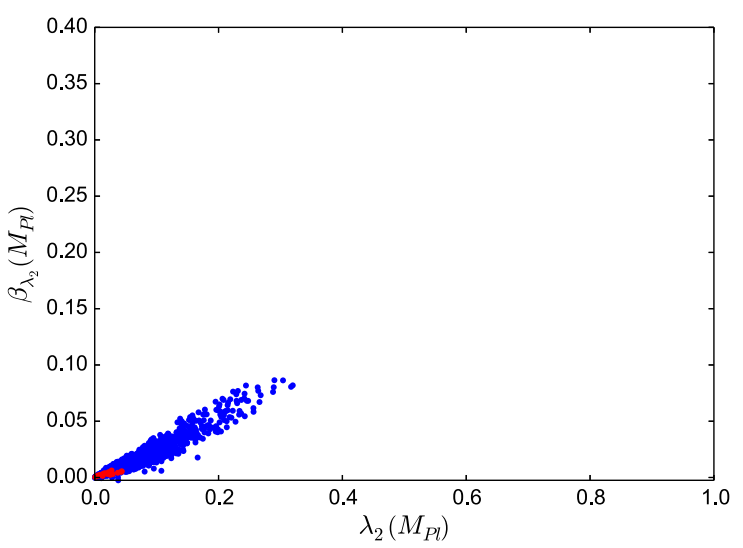

(b)

FIG. 3. Compatible values of the Higgs quartic coupling $\lambda_{2}\left(M_{\mathrm{Pl}}\right)$ against $\beta_{\lambda_{2}}\left(M_{\mathrm{Pl}}\right)$ in the type II 2HDM. (a) Includes points that are stable and perturbative up to $M_{\mathrm{Pl}}$ and includes an SM Higgs candidate, while (b) also enforces all relevant experimental constraints discussed in Sec. III. Blue points obey $\beta_{\lambda_{1,2,3,4}}<1.0$ at $M_{\mathrm{Pl}}$ while red points obey $\beta_{\lambda_{1}}<0.0127, \beta_{\lambda_{2}}<0.0064, \beta_{\lambda_{3}}<0.0139, \beta_{\lambda_{4}}<$ 0.0030 at $M_{\mathrm{Pl}}$.

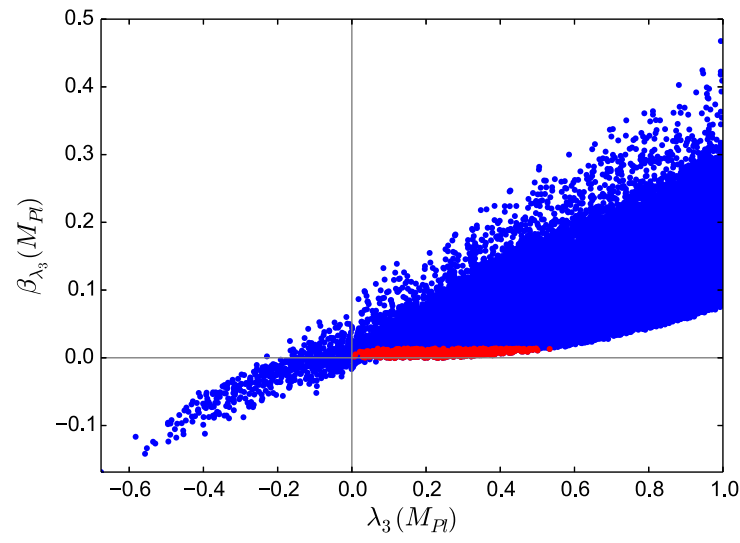

(a)

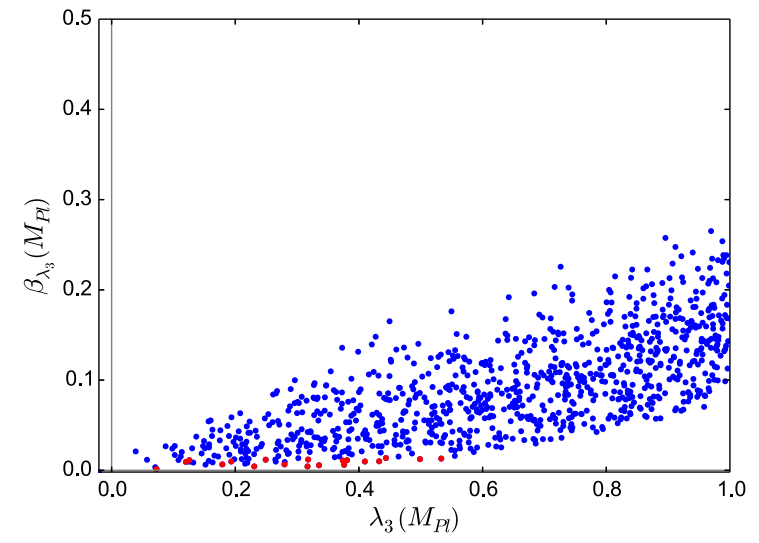

(b)

FIG. 4. Compatible values of the Higgs quartic coupling $\lambda_{3}\left(M_{\mathrm{Pl}}\right)$ against $\beta_{\lambda_{3}}\left(M_{\mathrm{Pl}}\right)$ in the type-II $2 \mathrm{HDM}$. (a) Includes points that are stable and perturbative up to $M_{\mathrm{Pl}}$ and includes an SM Higgs candidate, while (b) also enforces all relevant experimental constraints discussed in Sec. III. Blue points obey $\beta_{\lambda_{1,2,3,4}}<1.0$ at $M_{\mathrm{Pl}}$ while red points obey $\beta_{\lambda_{1}}<0.0127, \beta_{\lambda_{2}}<0.0064, \beta_{\lambda_{3}}<0.0139, \beta_{\lambda_{4}}<$ 0.0030 at $M_{\mathrm{Pl}}$.

$$
\begin{aligned}
\lambda_{5}(\Lambda) & =0, \\
\lambda_{4}(\Lambda) & <0, \\
\tilde{\lambda}(\Lambda)=\sqrt{\lambda_{1} \lambda_{2}}+\lambda_{3}+\min \left(0, \lambda_{4}\right) & =0, \\
\beta_{\tilde{\lambda}}(\Lambda) & =0,
\end{aligned}
$$

where the form of $\tilde{\lambda}$ arises from the minimization of the potential at $\Lambda$. We note that setting these conditions at $\Lambda$ results in a potential with more symmetry than the original $\mathbb{Z}_{2}$ symmetry of (2.3).

To investigate whether these MPP conditions in the type-II 2HDM are consistent with the current experimental constraints on the SM Higgs mass $m_{h}$ and the top pole mass $m_{t}$, we generated points in the parameter space as described in Sec. III, applying the theoretical constraint of vacuum stability at all scales. Figure 1(a) shows an example of the running of $\lambda_{1}, \lambda_{2}$, and $\tilde{\lambda}$ for a point that results in experimentally valid values of the SM Higgs mass and the top pole mass, and it is also consistent with the MPP conditions (4.1). Vacuum stability requires that all of these couplings remain greater than zero at all scales, but the running of $\tilde{\lambda}$ pulls it to negative values. Figure 1(b) shows values for the SM-like Higgs mass and top-quark mass arising from the new MPP boundary conditions, where red points correspond to choices with a stable potential and blue points to those that violate the stability conditions. Although there are many blue points with acceptable Higgs and top-quark masses, there are no satisfactory red points. Parameter choices that satisfy the vacuum stability conditions (red) have larger values of the top Yukawa $y_{t}$ which 


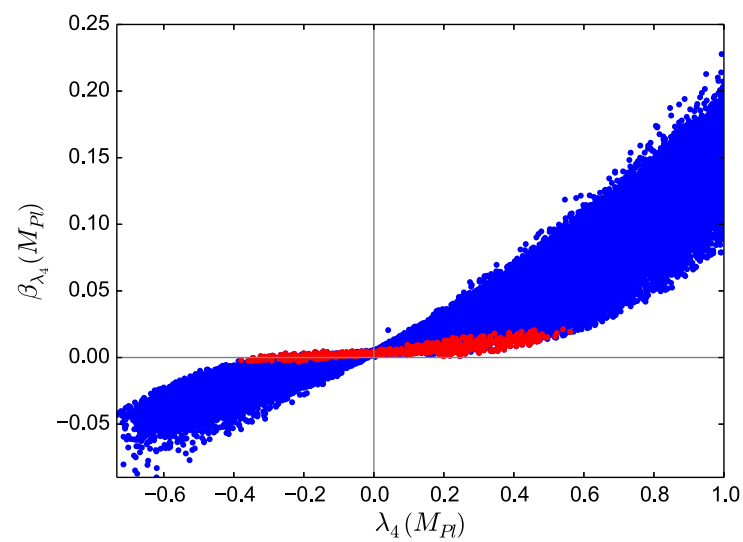

(a)

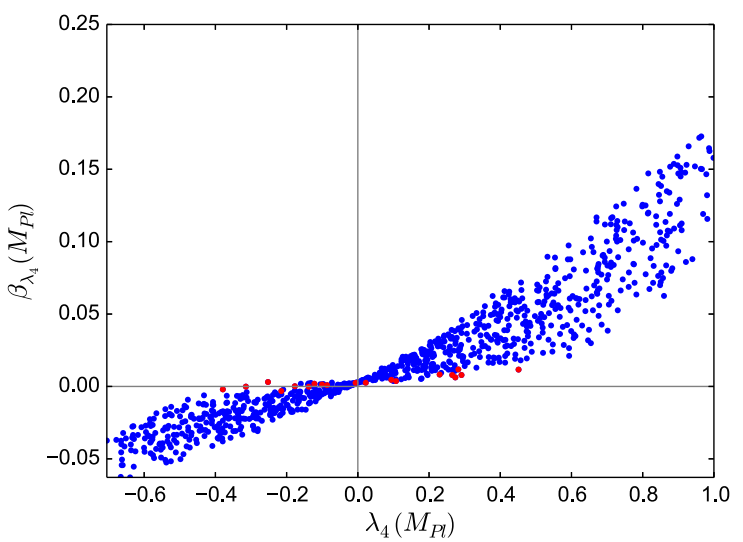

(b)

FIG. 5. Compatible values of the Higgs quartic coupling $\lambda_{4}\left(M_{\mathrm{Pl}}\right)$ against $\beta_{\lambda_{4}}\left(M_{\mathrm{Pl}}\right)$ in the type-II $2 \mathrm{HDM}$. (a) Includes points that are stable and perturbative up to $M_{\mathrm{Pl}}$ and includes an SM Higgs candidate, while (b) also enforces all relevant experimental constraints discussed in Sec. III. Blue points obey $\beta_{\lambda_{1,2,3,4}}<1.0$ at $M_{\mathrm{Pl}}$ while red points obey $\beta_{\lambda_{1}}<0.0127, \beta_{\lambda_{2}}<0.0064, \beta_{\lambda_{3}}<0.0139, \beta_{\lambda_{4}}<$ 0.0030 at $M_{\mathrm{Pl}}$.

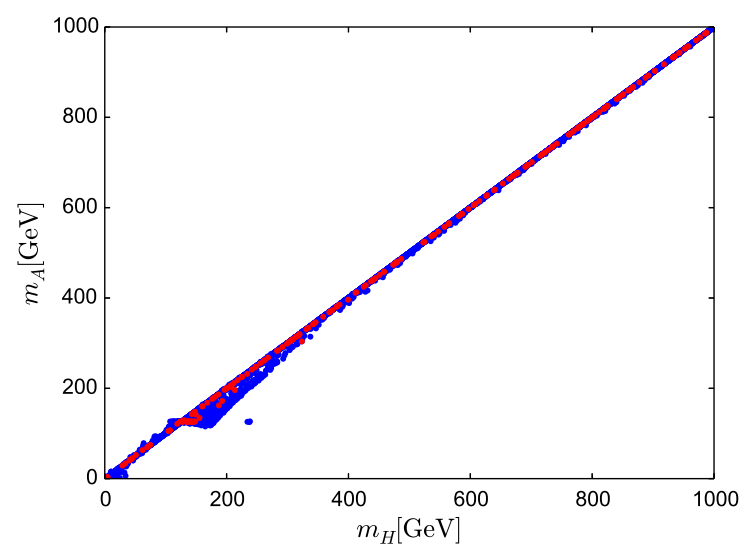

(a)

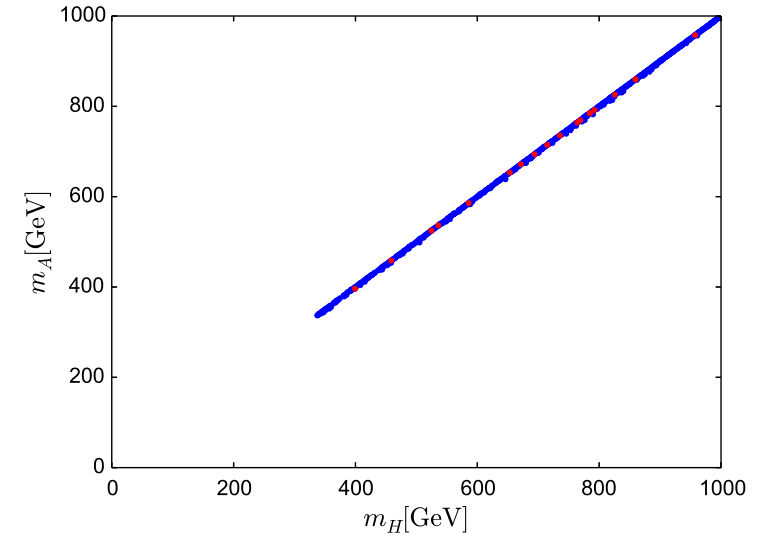

(b)

FIG. 6. Compatible values of the heavy neutral Higgs mass $m_{H}$ against the pseudoscalar Higgs $m_{A}$ in the type-II 2 HDM. (a) Includes points that are stable and perturbative up to $M_{\mathrm{Pl}}$ and includes an SM Higgs candidate, while (b) also enforces all relevant experimental constraints discussed in Sec. III. Blue points obey $\beta_{\lambda_{1,2,3,4}}<1.0$ at $M_{\mathrm{Pl}}$ while red points obey $\beta_{\lambda_{1}}<0.0127, \beta_{\lambda_{2}}<0.0064, \beta_{\lambda_{3}}<0.0139$, $\beta_{\lambda_{4}}<0.0030$ at $M_{\mathrm{Pl}}$.

positively contribute to the running of the quartic couplings. The larger required $y_{t}$ corresponds to a top mass in the range $220 \lesssim m_{t} \lesssim 230 \mathrm{GeV}$ which is not compatible with current experimental bounds on the top pole mass.

\section{B. Asymptotic safety in the type-II two Higgs doublet model}

Another possibility for the high scale dynamics that enforces high scale boundary conditions is asymptotic safety, in which the quartic couplings of the Higgs sector run toward an ultraviolet interacting fixed point [63-71]. It has been suggested that gravitational contributions may become significant at very high scales and alter the running of the couplings of the scalar potential to provide such a boundary condition [72-76]. In the context of the 2HDM, we are therefore seeking scenarios that exhibit zero values for the $\beta$ functions of the Higgs quartic couplings at the Planck scale while allowing the couplings themselves to be nonzero.

Note that it is important at this stage to be clear on what we mean by a $\beta$ function being zero. For each of the points in our parameter space we perform a perturbative calculation of the RGE evolution of the model couplings, and accommodate the uncertainty associated with this calculation by allowing for small, nonzero values of the $\beta$ functions. We estimate this uncertainty by using the difference between the $\beta$-function values at $M_{\mathrm{Pl}}$ calculated using one-loop and two-loop RGEs, and we consider the $\beta$ function to be zero if it is smaller than the RGE truncation error. In the case of the 2HDM we calculated, these are 


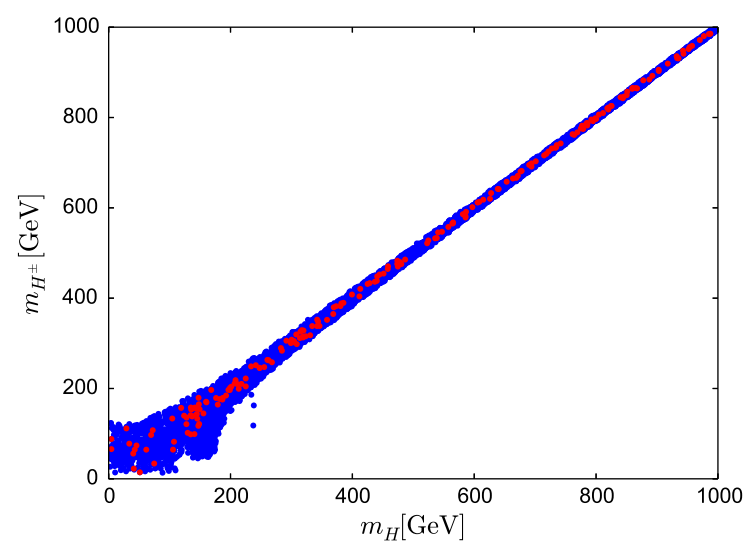

(a)

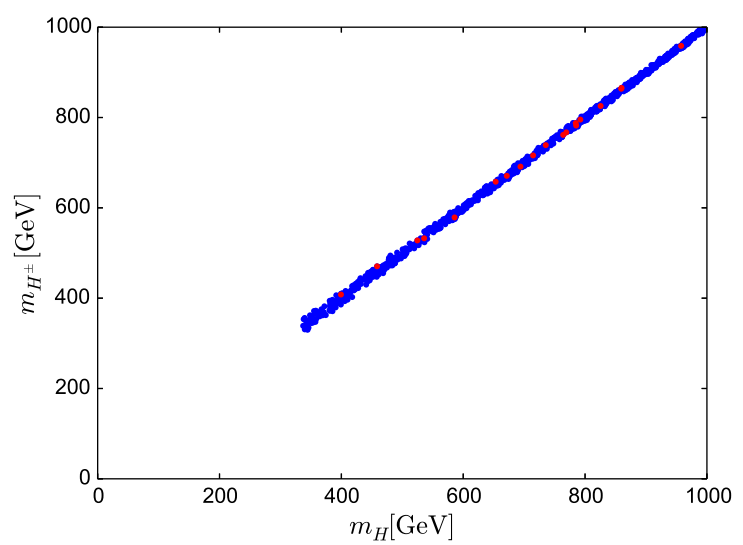

(b)

FIG. 7. Compatible values of the heavy neutral Higgs mass $m_{H}$ against the charged Higgs $m_{H^{ \pm}}$in the type-II 2HDM. (a) Includes points that are stable and perturbative up to $M_{\mathrm{Pl}}$ and includes an SM Higgs candidate, while (b) also enforces all relevant experimental constraints discussed in Sec. III. Blue points obey $\beta_{\lambda_{1,2,3,4}}<1.0$ at $M_{\mathrm{Pl}}$ while red points obey $\beta_{\lambda_{1}}<0.0127, \beta_{\lambda_{2}}<0.0064, \beta_{\lambda_{3}}<0.0139$, $\beta_{\lambda_{4}}<0.0030$ at $M_{\mathrm{Pl}}$.

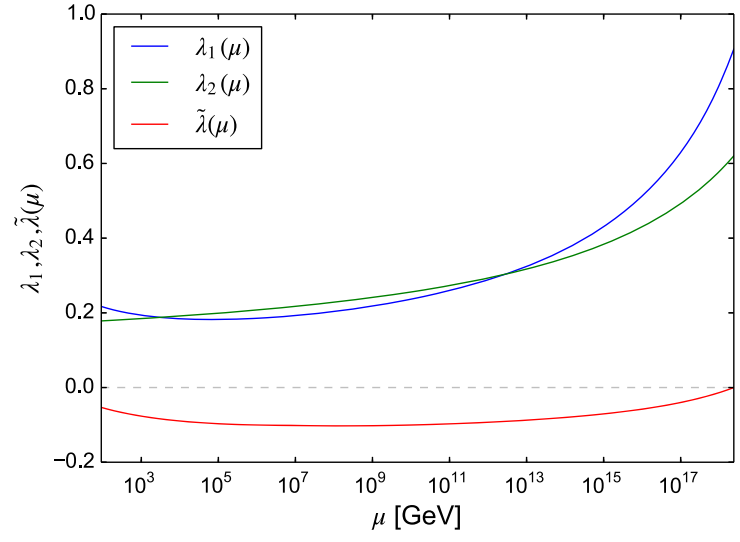

FIG. 8. Example running of $\lambda_{1}, \lambda_{2}$, and $\tilde{\lambda}$ for a point that provides valid masses for the SM Higgs and the top quark in the IDM. Boundedness from below and vacuum stability require that all three couplings are positive at all scales.

$$
\begin{aligned}
& \beta_{\lambda_{1}}\left(M_{\mathrm{Pl}}\right)<0.0127, \\
& \beta_{\lambda_{2}}\left(M_{\mathrm{Pl}}\right)<0.0064, \\
& \beta_{\lambda_{3}}\left(M_{\mathrm{Pl}}\right)<0.0139, \\
& \beta_{\lambda_{4}}\left(M_{\mathrm{Pl}}\right)<0.0030 .
\end{aligned}
$$

We now present the results of our numerical analysis of the type-II 2HDM, in which we look for regions of parameter space that are compatible with the high scale boundary conditions that can arise under the requirement for asymptotic safety. We apply the relevant theoretical and experimental constraints described in Sec. III as well as the $\beta_{\lambda_{i}}=0$ constraints shown in Eq. (4.2). Figures 2-5 show the values of the four nonzero quartic Higgs couplings $\lambda_{1,2,3,4}$ and their $\beta$ functions. The left plots include the theoretical constraints of perturbativity, vacuum stability, and a valid SM Higgs candidate, while those on the right also include experimental constraints. Points in red provide values of the $\beta$ functions that are compatible with our asymptotic safety high scale boundary conditions, while those in blue do not pass those constraints. Clearly there are regions of parameter space where all of the $\beta$ functions of the quartic Higgs couplings are within the truncation errors, even after all of the relevant experimental constraints have been applied. These regions correspond to very small but nonzero values of the quartic couplings at $M_{\mathrm{Pl}}$, consistent with a UV interacting fixed point.

Figure 6 shows the masses of the heavy neutral scalar $m_{H}$ against the pseudoscalar Higgs mass $m_{A}$, while Fig. 7 compares it with the charged Higgs mass $m_{H^{ \pm}}$. As the scale associated with the additional Higgs becomes significantly larger than the electroweak scale, the second doublet decouples from the first and the masses of $H, A$, and $H^{ \pm}$become degenerate. A lower limit on the masses of the extra scalars of around $m_{H, A, H^{ \pm}} \approx 330 \mathrm{GeV}$ is enforced once we apply the collider and flavor constraints. However, the points that are consistent with our high scale $\beta$ function conditions can have a range of different masses, and those conditions do not apply strong constraints upon the scalar mass spectrum in the type-II $2 \mathrm{HDM}$.

\section{The multiple point principle in the inert doublet model}

Equation (4.1) provides the conditions that a $2 \mathrm{HDM}$ parameter point must satisfy to be consistent with the MPP. These constraints also apply to the IDM. We examined the IDM parameter space in the same way as we did for the type-II $2 \mathrm{HDM}$ case detailed in Sec. IV A. We applied the MPP conditions at $M_{\mathrm{Pl}}$ and required valid points to be stable up to the Planck scale and to have a SM Higgs candidate. 


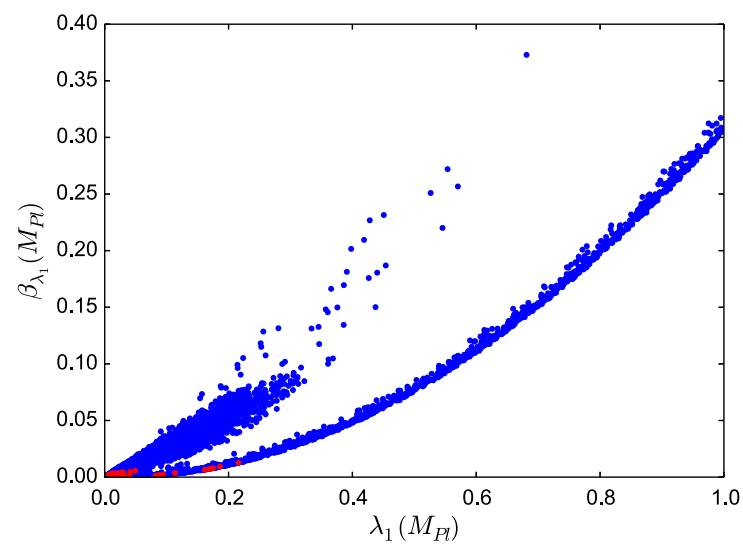

(a)

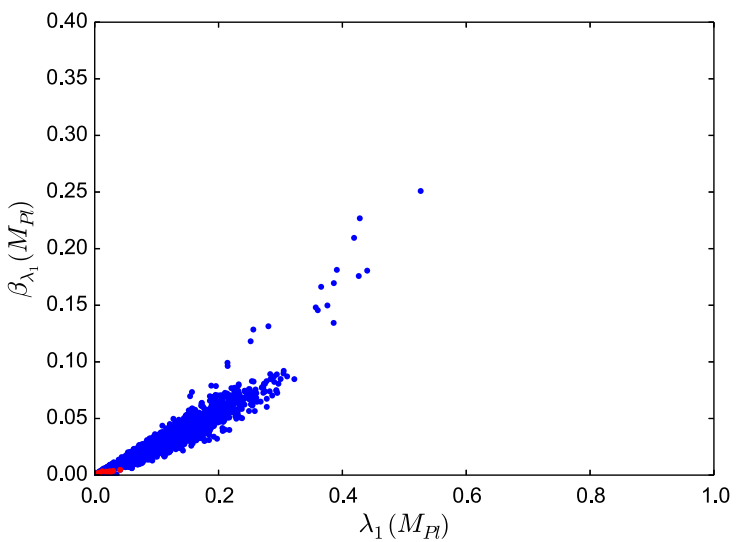

(b)

FIG. 9. Compatible values of the Higgs quartic coupling $\lambda_{1}\left(M_{\mathrm{Pl}}\right)$ against $\beta_{\lambda_{1}}\left(M_{\mathrm{Pl}}\right)$ in the IDM. (a) Includes points that are stable and perturbative up to $M_{\mathrm{Pl}}$ and includes an SM Higgs candidate, while (b) also enforces all relevant experimental constraints discussed in Sec. III. Blue points obey $\beta_{\lambda_{1,2,3,4}}<1.0$ at $M_{\mathrm{Pl}}$ while red points obey $\beta_{\lambda_{1}}<0.0127, \beta_{\lambda_{2}}<0.0064, \beta_{\lambda_{3}}<0.0139, \beta_{\lambda_{4}}<0.0030$ at $M_{\mathrm{Pl}}$.

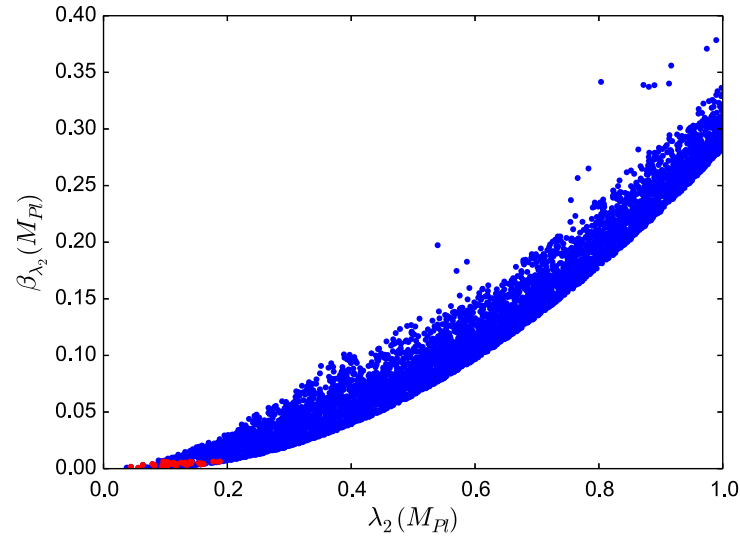

(a)

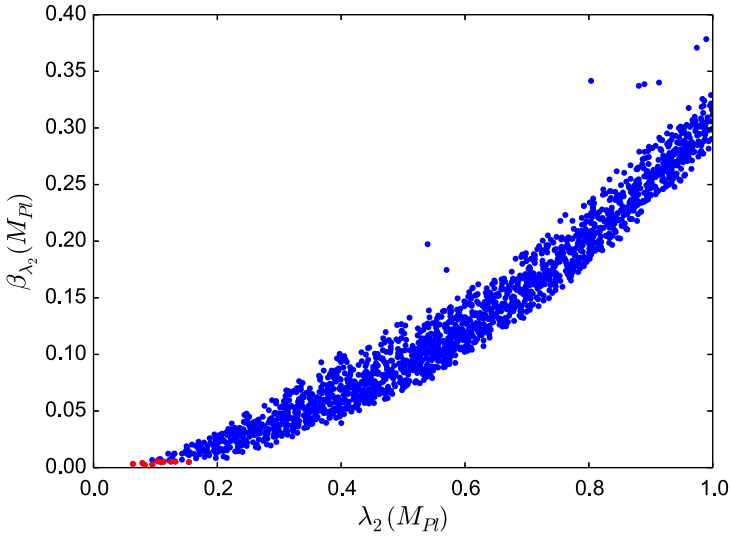

(b)

FIG. 10. Compatible values of the Higgs quartic coupling $\lambda_{2}\left(M_{\mathrm{Pl}}\right)$ against $\beta_{\lambda_{2}}\left(M_{\mathrm{Pl}}\right)$ in the IDM. (a) Includes points that are stable and perturbative up to $M_{\mathrm{Pl}}$ and includes an SM Higgs candidate, while (b) also enforces all relevant experimental constraints discussed in Sec. III. Blue points obey $\beta_{\lambda_{1,2,3,4}}<1.0$ at $M_{\mathrm{Pl}}$ while red points obey $\beta_{\lambda_{1}}<0.0127, \beta_{\lambda_{2}}<0.0064, \beta_{\lambda_{3}}<0.0139, \beta_{\lambda_{4}}<0.0030$ at $M_{\mathrm{Pl}}$.

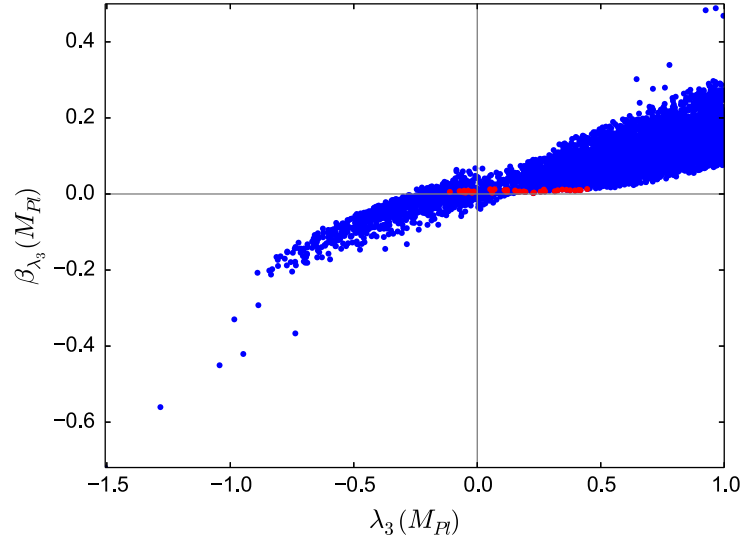

(a)

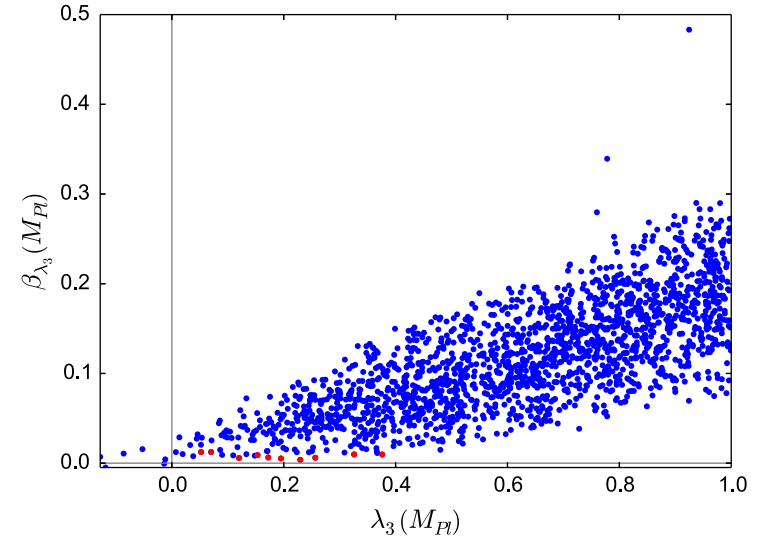

(b)

FIG. 11. Compatible values of the Higgs quartic coupling $\lambda_{3}\left(M_{\mathrm{Pl}}\right)$ against $\beta_{\lambda_{3}}\left(M_{\mathrm{Pl}}\right)$ in the IDM. (a) Includes points that are stable and perturbative up to $M_{\mathrm{Pl}}$ and includes an SM Higgs candidate, while (b) also enforces all relevant experimental constraints discussed in Sec. III. Blue points obey $\beta_{\lambda_{1,2,3,4}}<1.0$ at $M_{\mathrm{Pl}}$ while red points obey $\beta_{\lambda_{1}}<0.0127, \beta_{\lambda_{2}}<0.0064, \beta_{\lambda_{3}}<0.0139, \beta_{\lambda_{4}}<0.0030$ at $M_{\mathrm{Pl}}$. 


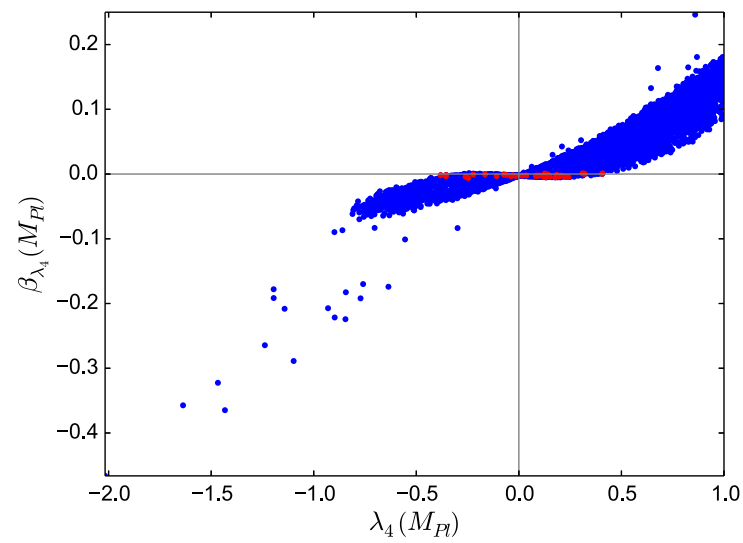

(a)

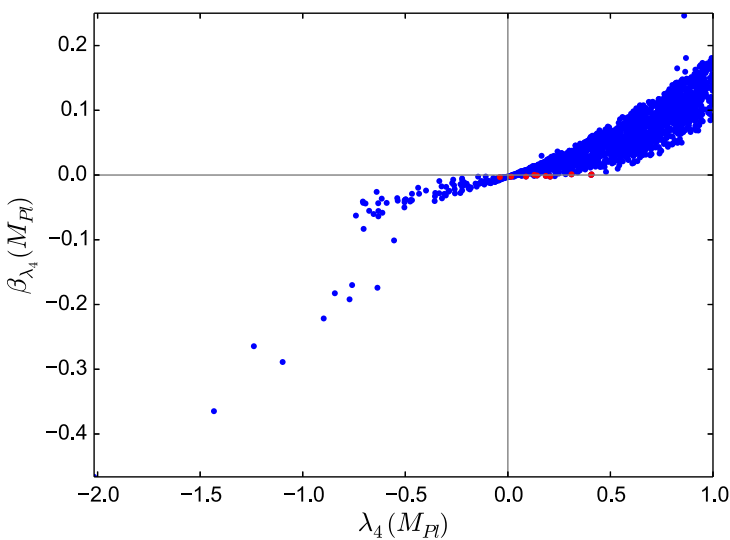

(b)

FIG. 12. Compatible values of the Higgs quartic coupling $\lambda_{4}\left(M_{\mathrm{Pl}}\right)$ against $\beta_{\lambda_{4}}\left(M_{\mathrm{Pl}}\right)$ in the IDM. (a) Includes points that are stable and perturbative up to $M_{\mathrm{Pl}}$ and includes an SM Higgs candidate, while (b) also enforces all relevant experimental constraints discussed in Sec. III. Blue points obey $\beta_{\lambda_{1,23.4}}<1.0$ at $M_{\mathrm{Pl}}$ while red points obey $\beta_{\lambda_{1}}<0.0127, \beta_{\lambda_{2}}<0.0064, \beta_{\lambda_{3}}<0.0139, \beta_{\lambda_{4}}<0.0030$ at $M_{\mathrm{Pl}}$.

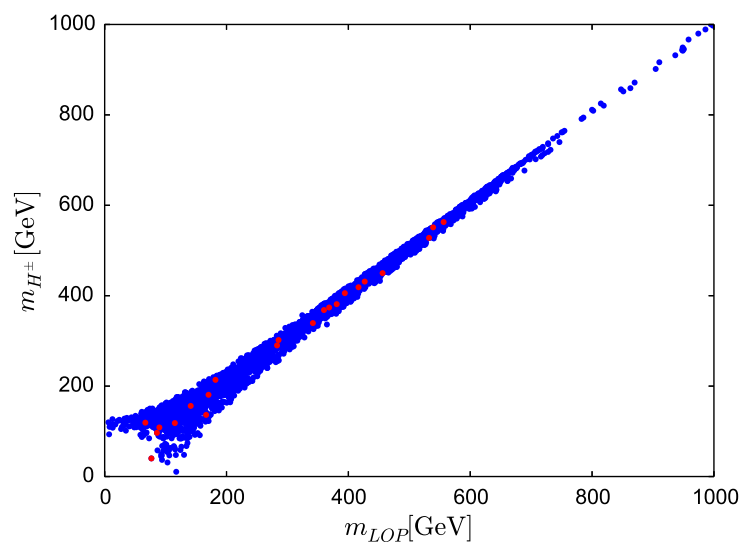

(a)

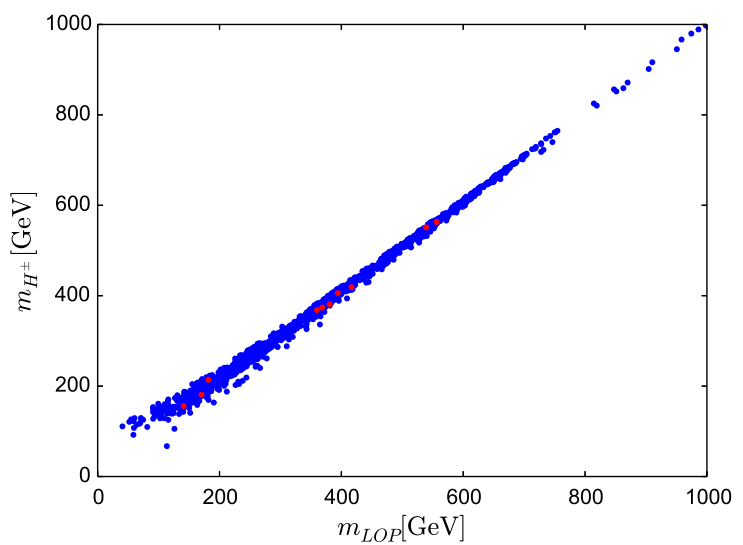

(b)

FIG. 13. Compatible values of the lightest odd particle mass $m_{\text {LOP }}$ against the charged Higgs mass $m_{H^{ \pm}}$in the IDM. (a) Includes points that are stable and perturbative up to $M_{\mathrm{Pl}}$ and includes an SM Higgs candidate, while (b) also enforces all relevant experimental constraints discussed in Sec. III. Blue points obey $\beta_{\lambda_{1,2.34}}<1.0$ at $M_{\mathrm{Pl}}$ while red points obey $\beta_{\lambda_{1}}<0.0127, \beta_{\lambda_{2}}<0.0064, \beta_{\lambda_{3}}<0.0139$, $\beta_{\lambda_{4}}<0.0030$ at $M_{\mathrm{Pl}}$.

Figure 8 shows the running of the quartic couplings $\lambda_{1}, \lambda_{2}$, and $\tilde{\lambda}$ for an example point in our scan that provided a valid SM Higgs and top mass. As in the type-II model, a stable vacuum requires all three of these couplings to be positive at all scales. Clearly this point fails our vacuum stability test and is representative of the other points in our scan. We found no points that could simultaneously satisfy the constraints of perturbativity, the vacuum stability, and the requirement of a realistic SM mass spectrum. Specifically, there are points that provide valid SM Higgs and top masses, but all of these points fail the condition $\tilde{\lambda}>0$. In fact, we found no points that could satisfy the MPP conditions outlined in Eq. (4.1) that remained stable up to the Planck scale, regardless of their Higgs or top masses.
This therefore suggests that the MPP cannot be implemented successfully in the IDM.

\section{Asymptotic safety in the inert doublet model}

We now present the results of our numerical analysis of the IDM. Figures 9-12 show points in the $\lambda_{i}-\beta_{\lambda_{i}}$ plane that satisfy both our theoretical and our experimental constraints as well as the asymptotic safety high scale boundary conditions of Eq. (4.2). The situation is somewhat similar to the type-II case discussed in Sec. IV B, inasmuch as there are points in the parameter space that are compatible with asymptotic safety and that those points have very small values of the quartic couplings. 
Figure 13 shows the allowed masses of the DM candidate $m_{\mathrm{LOP}}$ and the charged Higgs mass $m_{H^{ \pm}}$. The requirement that the LOP account for the DM relic density and the results from DM direct detection experiments places a lower limit on the LOP mass of $m_{\text {LOP }} \approx 40 \mathrm{GeV}$. As for the type-II case, points which meet the high scale constraint of asymptotic safety are seen to have a wide range of allowed scalar masses. It appears from our results that the existence of an interacting UV fixed point for the quartic couplings is valid under both the type-II model and the inert model. It places constraints on the high scale values of the quartic couplings, but due to the freedom to vary $m_{22}^{2}$ this does not translate to strong constraints on the possible masses of the new scalars.

\section{CONCLUSIONS}

We have investigated the type-II 2HDM and the IDM with a focus on possible constraints on the quartic Higgs couplings and their $\beta$ functions at the Planck scale. These high scale conditions may be a consequence of a second minimum in the potential that is degenerate with the electroweak minimum, as is the case in the MPP, or they may be due to the couplings running toward an interacting $\mathrm{UV}$ fixed point at $M_{\mathrm{Pl}}$, as for asymptotic safety. In this work we have examined the viability of these models with the required high scale boundary conditions, checking their compatibility with perturbativity, vacuum stability, and a SM Higgs candidate of the appropriate mass, as well as experimental constraints from colliders, flavor physics, and DM experiments.

Models with a second Higgs doublet have much more flexibility in their scalar potential than models with only one Higgs doublet, which gives them more freedom to accommodate the boundary conditions of the MPP or asymptotic safety. However, we found that both the type-II $2 \mathrm{HDM}$ and the IDM cannot satisfy the conditions required at the Planck scale by the MPP. Specifically, we found no points in either model's parameter space that was consistent with the MPP while also having a valid SM Higgs, an experimentally acceptable top quark mass, and a stable vacuum. In the type-II case we found that a stable vacuum would require a top mass on the order of $230 \mathrm{GeV}$, while in the inert case we found no points at all that could meet our theoretical requirements. The results of our analysis would suggest that the MPP is not compatible with the $2 \mathrm{HDM}$ or IDM that we investigated.
Asymptotic safety remains viable, as we found numerous points in the parameter space of both the 2HDM and the IDM that were compatible with theoretical and experimental constraints and had the required Planck scale values of the quartic Higgs $\beta$ functions. These points also have small but nonzero values of the corresponding quartic couplings, which is entirely in keeping with the existence of an interacting UV fixed point. The type-II case has a lower limit on the masses of the additional scalars of $m_{H, A, H^{ \pm}} \approx 330 \mathrm{GeV}$ imposed by experimental constraints. In the IDM the DM relic density and direct detection experiments place constraints on the mass of the model's DM candidate of $m_{\mathrm{LOP}} \approx 40 \mathrm{GeV}$. Although our investigation found regions of parameter space that are compatible with all constraints, they correspond to a range of masses for the extra Higgs, with no apparent restriction on those masses coming from the high scale boundary conditions.

Of course, the nonviability of the MPP for these two models does not imply that it is wrong. One could imagine additional matter being added to the model that could make such scenarios viable again. Additional matter added to the $2 \mathrm{HDM}$ or IDM would have the difficult task of forcing $\tilde{\lambda}$ to stay positive. However, it would be interesting to examine the SM Higgs sector with alternative additions, such as vectorlike fermions. Ultimately the question remains, is the peculiar behavior of the SM Higgs potential at the Planck scale a coincidence or a sign of new physics?

\section{ACKNOWLEDGMENTS}

The authors thank Peter Athron for invaluable help with FleXIBLESUSY, as well as Karl Nordstrom, António Morais, and David Sutherland for useful discussions. D. J. M. acknowledges partial support from the Science and Technology Facilities Council Grants No. ST/ L000446/1 and No. ST/P000746/1.

\section{APPENDIX: RENORMALIZATION GROUP EQUATIONS OF THE QUARTIC HIGGS COUPLINGS}

The two-loop $\beta$ functions that describe the running of the quartic Higgs couplings $\lambda_{1-7}$ are calculated using SARAH [26]. We present here the one- and two-loop contributions to $\beta_{\lambda_{i}}(i=1, \ldots, 7)$, that is $\beta_{\lambda_{i}}^{(1)}$ and $\beta_{\lambda_{i}}^{(2)}$, respectively; $g_{1-3}$ are the SM gauge couplings, and $Y_{f}(f=\{u, d, e\})$ are the Yukawa matrices:

$$
\begin{aligned}
\beta_{\lambda_{1}}^{(1)}= & +\frac{27}{200} g_{1}^{4}+\frac{9}{20} g_{1}^{2} g_{2}^{2}+\frac{9}{8} g_{2}^{4}-\frac{9}{5} g_{1}^{2} \lambda_{1}-9 g_{2}^{2} \lambda_{1}+24 \lambda_{1}^{2}+2 \lambda_{3}^{2}+2 \lambda_{3} \lambda_{4}+\lambda_{4}^{2}+\lambda_{5}^{2}+12 \lambda_{6}^{2} \\
& +12 \lambda_{1} \operatorname{Tr}\left(Y_{d} Y_{d}^{\dagger}\right)+4 \lambda_{1} \operatorname{Tr}\left(Y_{e} Y_{e}^{\dagger}\right)-6 \operatorname{Tr}\left(Y_{d} Y_{d}^{\dagger} Y_{d} Y_{d}^{\dagger}\right)-2 \operatorname{Tr}\left(Y_{e} Y_{e}^{\dagger} Y_{e} Y_{e}^{\dagger}\right),
\end{aligned}
$$




$$
\begin{aligned}
& \beta_{\lambda_{1}}^{(2)}=-\frac{3537}{2000} g_{1}^{6}-\frac{1719}{400} g_{1}^{4} g_{2}^{2}-\frac{303}{80} g_{1}^{2} g_{2}^{4}+\frac{291}{16} g_{2}^{6}+\frac{1953}{200} g_{1}^{4} \lambda_{1}+\frac{117}{20} g_{1}^{2} g_{2}^{2} \lambda_{1}-\frac{51}{8} g_{2}^{4} \lambda_{1} \\
& +\frac{108}{5} g_{1}^{2} \lambda_{1}^{2}+108 g_{2}^{2} \lambda_{1}^{2}-312 \lambda_{1}^{3}+\frac{9}{10} g_{1}^{4} \lambda_{3}+\frac{15}{2} g_{2}^{4} \lambda_{3}+\frac{12}{5} g_{1}^{2} \lambda_{3}^{2}+12 g_{2}^{2} \lambda_{3}^{2}-20 \lambda_{1} \lambda_{3}^{2}-8 \lambda_{3}^{3} \\
& +\frac{9}{20} g_{1}^{4} \lambda_{4}+\frac{3}{2} g_{1}^{2} g_{2}^{2} \lambda_{4}+\frac{15}{4} g_{2}^{4} \lambda_{4}+\frac{12}{5} g_{1}^{2} \lambda_{3} \lambda_{4}+12 g_{2}^{2} \lambda_{3} \lambda_{4}-20 \lambda_{1} \lambda_{3} \lambda_{4}-12 \lambda_{3}^{2} \lambda_{4}+\frac{6}{5} g_{1}^{2} \lambda_{4}^{2} \\
& +3 g_{2}^{2} \lambda_{4}^{2}-12 \lambda_{1} \lambda_{4}^{2}-16 \lambda_{3} \lambda_{4}^{2}-6 \lambda_{4}^{3}-\frac{3}{5} g_{1}^{2} \lambda_{5}^{2}-14 \lambda_{1} \lambda_{5}^{2}-20 \lambda_{3} \lambda_{5}^{2}-22 \lambda_{4} \lambda_{5}^{2} \\
& +\frac{54}{5} g_{1}^{2} \lambda_{6}^{2}+54 g_{2}^{2} \lambda_{6}^{2}-318 \lambda_{1} \lambda_{6}^{2}-66 \lambda_{3} \lambda_{6}^{2}-70 \lambda_{4} \lambda_{6}^{2}-74 \lambda_{5} \lambda_{6}^{2}-36 \lambda_{3} \lambda_{6} \lambda_{7} \\
& -28 \lambda_{4} \lambda_{6} \lambda_{7}-20 \lambda_{5} \lambda_{6} \lambda_{7}+6 \lambda_{1} \lambda_{7}^{2}-18 \lambda_{3} \lambda_{7}^{2}-14 \lambda_{4} \lambda_{7}^{2}-10 \lambda_{5} \lambda_{7}^{2} \\
& +\frac{1}{20}\left(-5\left(144 \lambda_{6}^{2}-320 g_{3}^{2} \lambda_{1}+576 \lambda_{1}^{2}-90 g_{2}^{2} \lambda_{1}+9 g_{2}^{4}\right)+9 g_{1}^{4}+g_{1}^{2}\left(50 \lambda_{1}+54 g_{2}^{2}\right)\right) \operatorname{Tr}\left(Y_{d} Y_{d}^{\dagger}\right) \\
& -\frac{3}{20}\left(15 g_{1}^{4}-2 g_{1}^{2}\left(11 g_{2}^{2}+25 \lambda_{1}\right)+5\left(-10 g_{2}^{2} \lambda_{1}+16 \lambda_{6}^{2}+64 \lambda_{1}^{2}+g_{2}^{4}\right)\right) \operatorname{Tr}\left(Y_{e} Y_{e}^{\dagger}\right) \\
& -12 \lambda_{3}^{2} \operatorname{Tr}\left(Y_{u} Y_{u}^{\dagger}\right)-12 \lambda_{3} \lambda_{4} \operatorname{Tr}\left(Y_{u} Y_{u}^{\dagger}\right)-6 \lambda_{4}^{2} \operatorname{Tr}\left(Y_{u} Y_{u}^{\dagger}\right)-6 \lambda_{5}^{2} \operatorname{Tr}\left(Y_{u} Y_{u}^{\dagger}\right)-36 \lambda_{6}^{2} \operatorname{Tr}\left(Y_{u} Y_{u}^{\dagger}\right) \\
& +\frac{4}{5} g_{1}^{2} \operatorname{Tr}\left(Y_{d} Y_{d}^{\dagger} Y_{d} Y_{d}^{\dagger}\right)-32 g_{3}^{2} \operatorname{Tr}\left(Y_{d} Y_{d}^{\dagger} Y_{d} Y_{d}^{\dagger}\right)-3 \lambda_{1} \operatorname{Tr}\left(Y_{d} Y_{d}^{\dagger} Y_{d} Y_{d}^{\dagger}\right)-9 \lambda_{1} \operatorname{Tr}\left(Y_{d} Y_{u}^{\dagger} Y_{u} Y_{d}^{\dagger}\right) \\
& -\frac{12}{5} g_{1}^{2} \operatorname{Tr}\left(Y_{e} Y_{e}^{\dagger} Y_{e} Y_{e}^{\dagger}\right)-\lambda_{1} \operatorname{Tr}\left(Y_{e} Y_{e}^{\dagger} Y_{e} Y_{e}^{\dagger}\right)+30 \operatorname{Tr}\left(Y_{d} Y_{d}^{\dagger} Y_{d} Y_{d}^{\dagger} Y_{d} Y_{d}^{\dagger}\right)+6 \operatorname{Tr}\left(Y_{d} Y_{u}^{\dagger} Y_{u} Y_{d}^{\dagger} Y_{d} Y_{d}^{\dagger}\right) \\
& \beta_{\lambda_{2}}^{(1)}=+\frac{27}{200} g_{1}^{4}+\frac{9}{20} g_{1}^{2} g_{2}^{2}+\frac{9}{8} g_{2}^{4}-\frac{9}{5} g_{1}^{2} \lambda_{2}-9 g_{2}^{2} \lambda_{2}+24 \lambda_{2}^{2}+2 \lambda_{3}^{2}+2 \lambda_{3} \lambda_{4}+\lambda_{4}^{2}+\lambda_{5}^{2}+12 \lambda_{7}^{2} \\
& +12 \lambda_{2} \operatorname{Tr}\left(Y_{u} Y_{u}^{\dagger}\right)-6 \operatorname{Tr}\left(Y_{u} Y_{u}^{\dagger} Y_{u} Y_{u}^{\dagger}\right) \\
& \beta_{\lambda_{2}}^{(2)}=-\frac{3537}{2000} g_{1}^{6}-\frac{1719}{400} g_{1}^{4} g_{2}^{2}-\frac{303}{80} g_{1}^{2} g_{2}^{4}+\frac{291}{16} g_{2}^{6}+\frac{1953}{200} g_{1}^{4} \lambda_{2}+\frac{117}{20} g_{1}^{2} g_{2}^{2} \lambda_{2}-\frac{51}{8} g_{2}^{4} \lambda_{2}+\frac{108}{5} g_{1}^{2} \lambda_{2}^{2} \\
& +108 g_{2}^{2} \lambda_{2}^{2}-312 \lambda_{2}^{3}+\frac{9}{10} g_{1}^{4} \lambda_{3}+\frac{15}{2} g_{2}^{4} \lambda_{3}+\frac{12}{5} g_{1}^{2} \lambda_{3}^{2}+12 g_{2}^{2} \lambda_{3}^{2}-20 \lambda_{2} \lambda_{3}^{2}-8 \lambda_{3}^{3}+\frac{9}{20} g_{1}^{4} \lambda_{4} \\
& +\frac{3}{2} g_{1}^{2} g_{2}^{2} \lambda_{4}+\frac{15}{4} g_{2}^{4} \lambda_{4}+\frac{12}{5} g_{1}^{2} \lambda_{3} \lambda_{4}+12 g_{2}^{2} \lambda_{3} \lambda_{4}-20 \lambda_{2} \lambda_{3} \lambda_{4}-12 \lambda_{3}^{2} \lambda_{4}+\frac{6}{5} g_{1}^{2} \lambda_{4}^{2} \\
& +3 g_{2}^{2} \lambda_{4}^{2}-12 \lambda_{2} \lambda_{4}^{2}-16 \lambda_{3} \lambda_{4}^{2}-6 \lambda_{4}^{3}-\frac{3}{5} g_{1}^{2} \lambda_{5}^{2}-14 \lambda_{2} \lambda_{5}^{2}-20 \lambda_{3} \lambda_{5}^{2}-22 \lambda_{4} \lambda_{5}^{2}+6 \lambda_{2} \lambda_{6}^{2} \\
& -18 \lambda_{3} \lambda_{6}^{2}-14 \lambda_{4} \lambda_{6}^{2}-10 \lambda_{5} \lambda_{6}^{2}-36 \lambda_{3} \lambda_{6} \lambda_{7}-28 \lambda_{4} \lambda_{6} \lambda_{7}-20 \lambda_{5} \lambda_{6} \lambda_{7}+\frac{54}{5} g_{1}^{2} \lambda_{7}^{2}+54 g_{2}^{2} \lambda_{7}^{2} \\
& -318 \lambda_{2} \lambda_{7}^{2}-66 \lambda_{3} \lambda_{7}^{2}-70 \lambda_{4} \lambda_{7}^{2}-74 \lambda_{5} \lambda_{7}^{2}-6\left(2 \lambda_{3}^{2}+2 \lambda_{3} \lambda_{4}+6 \lambda_{7}^{2}+\lambda_{4}^{2}+\lambda_{5}^{2}\right) \operatorname{Tr}\left(Y_{d} Y_{d}^{\dagger}\right) \\
& -2\left(2 \lambda_{3}^{2}+2 \lambda_{3} \lambda_{4}+6 \lambda_{7}^{2}+\lambda_{4}^{2}+\lambda_{5}^{2}\right) \operatorname{Tr}\left(Y_{e} Y_{e}^{\dagger}\right)-\frac{171}{100} g_{1}^{4} \operatorname{Tr}\left(Y_{u} Y_{u}^{\dagger}\right)+\frac{63}{10} g_{1}^{2} g_{2}^{2} \operatorname{Tr}\left(Y_{u} Y_{u}^{\dagger}\right) \\
& -\frac{9}{4} g_{2}^{4} \operatorname{Tr}\left(Y_{u} Y_{u}^{\dagger}\right)+\frac{17}{2} g_{1}^{2} \lambda_{2} \operatorname{Tr}\left(Y_{u} Y_{u}^{\dagger}\right)+\frac{45}{2} g_{2}^{2} \lambda_{2} \operatorname{Tr}\left(Y_{u} Y_{u}^{\dagger}\right)+80 g_{3}^{2} \lambda_{2} \operatorname{Tr}\left(Y_{u} Y_{u}^{\dagger}\right) \\
& -144 \lambda_{2}^{2} \operatorname{Tr}\left(Y_{u} Y_{u}^{\dagger}\right)-36 \lambda_{7}^{2} \operatorname{Tr}\left(Y_{u} Y_{u}^{\dagger}\right)-9 \lambda_{2} \operatorname{Tr}\left(Y_{d} Y_{u}^{\dagger} Y_{u} Y_{d}^{\dagger}\right)-\frac{8}{5} g_{1}^{2} \operatorname{Tr}\left(Y_{u} Y_{u}^{\dagger} Y_{u} Y_{u}^{\dagger}\right) \\
& -32 g_{3}^{2} \operatorname{Tr}\left(Y_{u} Y_{u}^{\dagger} Y_{u} Y_{u}^{\dagger}\right)-3 \lambda_{2} \operatorname{Tr}\left(Y_{u} Y_{u}^{\dagger} Y_{u} Y_{u}^{\dagger}\right)+6 \operatorname{Tr}\left(Y_{d} Y_{u}^{\dagger} Y_{u} Y_{u}^{\dagger} Y_{u} Y_{d}^{\dagger}\right) \\
& +30 \operatorname{Tr}\left(Y_{u} Y_{u}^{\dagger} Y_{u} Y_{u}^{\dagger} Y_{u} Y_{u}^{\dagger}\right)+10 \operatorname{Tr}\left(Y_{e} Y_{e}^{\dagger} Y_{e} Y_{e}^{\dagger} Y_{e} Y_{e}^{\dagger}\right),
\end{aligned}
$$




$$
\begin{aligned}
& \beta_{\lambda_{3}}^{(1)}=+\frac{27}{100} g_{1}^{4}-\frac{9}{10} g_{1}^{2} g_{2}^{2}+\frac{9}{4} g_{2}^{4}-\frac{9}{5} g_{1}^{2} \lambda_{3}-9 g_{2}^{2} \lambda_{3}+12 \lambda_{1} \lambda_{3}+12 \lambda_{2} \lambda_{3}+4 \lambda_{3}^{2}+4 \lambda_{1} \lambda_{4} \\
& +4 \lambda_{2} \lambda_{4}+2 \lambda_{4}^{2}+2 \lambda_{5}^{2}+4 \lambda_{6}^{2}+16 \lambda_{6} \lambda_{7}+4 \lambda_{7}^{2}+6 \lambda_{3} \operatorname{Tr}\left(Y_{d} Y_{d}^{\dagger}\right)+2 \lambda_{3} \operatorname{Tr}\left(Y_{e} Y_{e}^{\dagger}\right) \\
& +6 \lambda_{3} \operatorname{Tr}\left(Y_{u} Y_{u}^{\dagger}\right)-12 \operatorname{Tr}\left(Y_{d} Y_{u}^{\dagger} Y_{u} Y_{d}^{\dagger}\right) \text {, } \\
& \beta_{\lambda_{3}}^{(2)}=-\frac{3537}{1000} g_{1}^{6}+\frac{909}{200} g_{1}^{4} g_{2}^{2}+\frac{33}{40} g_{1}^{2} g_{2}^{4}+\frac{291}{8} g_{2}^{6}+\frac{27}{10} g_{1}^{4} \lambda_{1}-3 g_{1}^{2} g_{2}^{2} \lambda_{1}+\frac{45}{2} g_{2}^{4} \lambda_{1}+\frac{27}{10} g_{1}^{4} \lambda_{2} \\
& -3 g_{1}^{2} g_{2}^{2} \lambda_{2}+\frac{45}{2} g_{2}^{4} \lambda_{2}+\frac{1773}{200} g_{1}^{4} \lambda_{3}+\frac{33}{20} g_{1}^{2} g_{2}^{2} \lambda_{3}-\frac{111}{8} g_{2}^{4} \lambda_{3}+\frac{72}{5} g_{1}^{2} \lambda_{1} \lambda_{3}+72 g_{2}^{2} \lambda_{1} \lambda_{3} \\
& -60 \lambda_{1}^{2} \lambda_{3}+\frac{72}{5} g_{1}^{2} \lambda_{2} \lambda_{3}+72 g_{2}^{2} \lambda_{2} \lambda_{3}-60 \lambda_{2}^{2} \lambda_{3}+\frac{6}{5} g_{1}^{2} \lambda_{3}^{2}+6 g_{2}^{2} \lambda_{3}^{2}-72 \lambda_{1} \lambda_{3}^{2}-72 \lambda_{2} \lambda_{3}^{2} \\
& -12 \lambda_{3}^{3}+\frac{9}{10} g_{1}^{4} \lambda_{4}-\frac{9}{5} g_{1}^{2} g_{2}^{2} \lambda_{4}+\frac{15}{2} g_{2}^{4} \lambda_{4}+\frac{24}{5} g_{1}^{2} \lambda_{1} \lambda_{4}+36 g_{2}^{2} \lambda_{1} \lambda_{4}-16 \lambda_{1}^{2} \lambda_{4}+\frac{24}{5} g_{1}^{2} \lambda_{2} \lambda_{4} \\
& +36 g_{2}^{2} \lambda_{2} \lambda_{4}-16 \lambda_{2}^{2} \lambda_{4}-12 g_{2}^{2} \lambda_{3} \lambda_{4}-32 \lambda_{1} \lambda_{3} \lambda_{4}-32 \lambda_{2} \lambda_{3} \lambda_{4}-4 \lambda_{3}^{2} \lambda_{4}-\frac{6}{5} g_{1}^{2} \lambda_{4}^{2} \\
& +6 g_{2}^{2} \lambda_{4}^{2}-28 \lambda_{1} \lambda_{4}^{2}-28 \lambda_{2} \lambda_{4}^{2}-16 \lambda_{3} \lambda_{4}^{2}-12 \lambda_{4}^{3}+\frac{12}{5} g_{1}^{2} \lambda_{5}^{2}-36 \lambda_{1} \lambda_{5}^{2}-36 \lambda_{2} \lambda_{5}^{2} \\
& -18 \lambda_{3} \lambda_{5}^{2}-44 \lambda_{4} \lambda_{5}^{2}+\frac{6}{5} g_{1}^{2} \lambda_{6}^{2}-124 \lambda_{1} \lambda_{6}^{2}-44 \lambda_{2} \lambda_{6}^{2}-60 \lambda_{3} \lambda_{6}^{2}-68 \lambda_{4} \lambda_{6}^{2}-68 \lambda_{5} \lambda_{6}^{2} \\
& +\frac{96}{5} g_{1}^{2} \lambda_{6} \lambda_{7}+108 g_{2}^{2} \lambda_{6} \lambda_{7}-88 \lambda_{1} \lambda_{6} \lambda_{7}-88 \lambda_{2} \lambda_{6} \lambda_{7}-176 \lambda_{3} \lambda_{6} \lambda_{7}-88 \lambda_{4} \lambda_{6} \lambda_{7} \\
& -72 \lambda_{5} \lambda_{6} \lambda_{7}+\frac{6}{5} g_{1}^{2} \lambda_{7}^{2}-44 \lambda_{1} \lambda_{7}^{2}-124 \lambda_{2} \lambda_{7}^{2}-60 \lambda_{3} \lambda_{7}^{2}-68 \lambda_{4} \lambda_{7}^{2}-68 \lambda_{5} \lambda_{7}^{2} \\
& +\frac{1}{20}\left(9 g_{1}^{4}+g_{1}^{2}\left(25 \lambda_{3}-54 g_{2}^{2}\right)-5\left(-45 g_{2}^{2} \lambda_{3}\right.\right. \\
& \left.\left.+8\left(-20 g_{3}^{2} \lambda_{3}+3\left(2 \lambda_{3}^{2}+4 \lambda_{1}\left(3 \lambda_{3}+\lambda_{4}\right)+4 \lambda_{6}^{2}+8 \lambda_{6} \lambda_{7}+\lambda_{4}^{2}+\lambda_{5}^{2}\right)\right)+9 g_{2}^{4}\right)\right) \operatorname{Tr}\left(Y_{d} Y_{d}^{\dagger}\right) \\
& -\frac{1}{20}\left(45 g_{1}^{4}+5\left(-15 g_{2}^{2} \lambda_{3}+3 g_{2}^{4}+8\left(2 \lambda_{3}^{2}+4 \lambda_{1}\left(3 \lambda_{3}+\lambda_{4}\right)+4 \lambda_{6}^{2}+8 \lambda_{6} \lambda_{7}+\lambda_{4}^{2}+\lambda_{5}^{2}\right)\right)\right. \\
& \left.+g_{1}^{2}\left(66 g_{2}^{2}-75 \lambda_{3}\right)\right) \operatorname{Tr}\left(Y_{e} Y_{e}^{\dagger}\right)-\frac{171}{100} g_{1}^{4} \operatorname{Tr}\left(Y_{u} Y_{u}^{\dagger}\right)-\frac{63}{10} g_{1}^{2} g_{2}^{2} \operatorname{Tr}\left(Y_{u} Y_{u}^{\dagger}\right)-\frac{9}{4} g_{2}^{4} \operatorname{Tr}\left(Y_{u} Y_{u}^{\dagger}\right) \\
& +\frac{17}{4} g_{1}^{2} \lambda_{3} \operatorname{Tr}\left(Y_{u} Y_{u}^{\dagger}\right)+\frac{45}{4} g_{2}^{2} \lambda_{3} \operatorname{Tr}\left(Y_{u} Y_{u}^{\dagger}\right)+40 g_{3}^{2} \lambda_{3} \operatorname{Tr}\left(Y_{u} Y_{u}^{\dagger}\right)-72 \lambda_{2} \lambda_{3} \operatorname{Tr}\left(Y_{u} Y_{u}^{\dagger}\right) \\
& -12 \lambda_{3}^{2} \operatorname{Tr}\left(Y_{u} Y_{u}^{\dagger}\right)-24 \lambda_{2} \lambda_{4} \operatorname{Tr}\left(Y_{u} Y_{u}^{\dagger}\right)-6 \lambda_{4}^{2} \operatorname{Tr}\left(Y_{u} Y_{u}^{\dagger}\right)-6 \lambda_{5}^{2} \operatorname{Tr}\left(Y_{u} Y_{u}^{\dagger}\right) \\
& -48 \lambda_{6} \lambda_{7} \operatorname{Tr}\left(Y_{u} Y_{u}^{\dagger}\right)-24 \lambda_{7}^{2} \operatorname{Tr}\left(Y_{u} Y_{u}^{\dagger}\right)-\frac{27}{2} \lambda_{3} \operatorname{Tr}\left(Y_{d} Y_{d}^{\dagger} Y_{d} Y_{d}^{\dagger}\right)-\frac{4}{5} g_{1}^{2} \operatorname{Tr}\left(Y_{d} Y_{u}^{\dagger} Y_{u} Y_{d}^{\dagger}\right) \\
& -64 g_{3}^{2} \operatorname{Tr}\left(Y_{d} Y_{u}^{\dagger} Y_{u} Y_{d}^{\dagger}\right)+15 \lambda_{3} \operatorname{Tr}\left(Y_{d} Y_{u}^{\dagger} Y_{u} Y_{d}^{\dagger}\right)-\frac{9}{2} \lambda_{3} \operatorname{Tr}\left(Y_{e} Y_{e}^{\dagger} Y_{e} Y_{e}^{\dagger}\right) \\
& -\frac{27}{2} \lambda_{3} \operatorname{Tr}\left(Y_{u} Y_{u}^{\dagger} Y_{u} Y_{u}^{\dagger}\right)+12 \operatorname{Tr}\left(Y_{d} Y_{d}^{\dagger} Y_{d} Y_{u}^{\dagger} Y_{u} Y_{d}^{\dagger}\right)+24 \operatorname{Tr}\left(Y_{d} Y_{u}^{\dagger} Y_{u} Y_{d}^{\dagger} Y_{d} Y_{d}^{\dagger}\right) \\
& +36 \operatorname{Tr}\left(Y_{d} Y_{u}^{\dagger} Y_{u} Y_{u}^{\dagger} Y_{u} Y_{d}^{\dagger}\right) \text {, } \\
& \beta_{\lambda_{4}}^{(1)}=+\frac{9}{5} g_{1}^{2} g_{2}^{2}-\frac{9}{5} g_{1}^{2} \lambda_{4}-9 g_{2}^{2} \lambda_{4}+4 \lambda_{1} \lambda_{4}+4 \lambda_{2} \lambda_{4}+8 \lambda_{3} \lambda_{4}+4 \lambda_{4}^{2}+8 \lambda_{5}^{2}+10 \lambda_{6}^{2}+4 \lambda_{6} \lambda_{7}+10 \lambda_{7}^{2} \\
& +6 \lambda_{4} \operatorname{Tr}\left(Y_{d} Y_{d}^{\dagger}\right)+2 \lambda_{4} \operatorname{Tr}\left(Y_{e} Y_{e}^{\dagger}\right)+6 \lambda_{4} \operatorname{Tr}\left(Y_{u} Y_{u}^{\dagger}\right)+12 \operatorname{Tr}\left(Y_{d} Y_{u}^{\dagger} Y_{u} Y_{d}^{\dagger}\right),
\end{aligned}
$$




$$
\begin{aligned}
& \beta_{\lambda_{4}}^{(2)}=-\frac{657}{50} g_{1}^{4} g_{2}^{2}-\frac{42}{5} g_{1}^{2} g_{2}^{4}+6 g_{1}^{2} g_{2}^{2} \lambda_{1}+6 g_{1}^{2} g_{2}^{2} \lambda_{2}+\frac{6}{5} g_{1}^{2} g_{2}^{2} \lambda_{3}+\frac{1413}{200} g_{1}^{4} \lambda_{4}+\frac{153}{20} g_{1}^{2} g_{2}^{2} \lambda_{4} \\
& -\frac{231}{8} g_{2}^{4} \lambda_{4}+\frac{24}{5} g_{1}^{2} \lambda_{1} \lambda_{4}-28 \lambda_{1}^{2} \lambda_{4}+\frac{24}{5} g_{1}^{2} \lambda_{2} \lambda_{4}-28 \lambda_{2}^{2} \lambda_{4}+\frac{12}{5} g_{1}^{2} \lambda_{3} \lambda_{4}+36 g_{2}^{2} \lambda_{3} \lambda_{4} \\
& -80 \lambda_{1} \lambda_{3} \lambda_{4}-80 \lambda_{2} \lambda_{3} \lambda_{4}-28 \lambda_{3}^{2} \lambda_{4}+\frac{24}{5} g_{1}^{2} \lambda_{4}^{2}+18 g_{2}^{2} \lambda_{4}^{2}-40 \lambda_{1} \lambda_{4}^{2}-40 \lambda_{2} \lambda_{4}^{2}-28 \lambda_{3} \lambda_{4}^{2} \\
& +\frac{48}{5} g_{1}^{2} \lambda_{5}^{2}+54 g_{2}^{2} \lambda_{5}^{2}-48 \lambda_{1} \lambda_{5}^{2}-48 \lambda_{2} \lambda_{5}^{2}-48 \lambda_{3} \lambda_{5}^{2}-26 \lambda_{4} \lambda_{5}^{2}+\frac{42}{5} g_{1}^{2} \lambda_{6}^{2}+54 g_{2}^{2} \lambda_{6}^{2} \\
& -148 \lambda_{1} \lambda_{6}^{2}-20 \lambda_{2} \lambda_{6}^{2}-72 \lambda_{3} \lambda_{6}^{2}-68 \lambda_{4} \lambda_{6}^{2}-80 \lambda_{5} \lambda_{6}^{2}+\frac{24}{5} g_{1}^{2} \lambda_{6} \lambda_{7}-40 \lambda_{1} \lambda_{6} \lambda_{7}-40 \lambda_{2} \lambda_{6} \lambda_{7} \\
& -80 \lambda_{3} \lambda_{6} \lambda_{7}-160 \lambda_{4} \lambda_{6} \lambda_{7}-96 \lambda_{5} \lambda_{6} \lambda_{7}+\frac{42}{5} g_{1}^{2} \lambda_{7}^{2}+54 g_{2}^{2} \lambda_{7}^{2}-20 \lambda_{1} \lambda_{7}^{2}-148 \lambda_{2} \lambda_{7}^{2} \\
& -72 \lambda_{3} \lambda_{7}^{2}-68 \lambda_{4} \lambda_{7}^{2}-80 \lambda_{5} \lambda_{7}^{2}+\frac{1}{20}\left(5 \left(1 6 \left(10 g_{3}^{2} \lambda_{4}-3\left(2 \lambda_{1} \lambda_{4}+2 \lambda_{3} \lambda_{4}+2 \lambda_{5}^{2}\right.\right.\right.\right. \\
& \left.\left.\left.\left.+5 \lambda_{6}^{2}+\lambda_{6} \lambda_{7}+\lambda_{4}^{2}\right)\right)+45 g_{2}^{2} \lambda_{4}\right)+g_{1}^{2}\left(108 g_{2}^{2}+25 \lambda_{4}\right)\right) \operatorname{Tr}\left(Y_{d} Y_{d}^{\dagger}\right)+\frac{1}{20}\left(3 g _ { 1 } ^ { 2 } \left(25 \lambda_{4}\right.\right. \\
& \left.\left.+44 g_{2}^{2}\right)+5\left(15 g_{2}^{2} \lambda_{4}-16\left(2 \lambda_{1} \lambda_{4}+2 \lambda_{3} \lambda_{4}+2 \lambda_{5}^{2}+5 \lambda_{6}^{2}+\lambda_{6} \lambda_{7}+\lambda_{4}^{2}\right)\right)\right) \operatorname{Tr}\left(Y_{e} Y_{e}^{\dagger}\right) \\
& +\frac{63}{5} g_{1}^{2} g_{2}^{2} \operatorname{Tr}\left(Y_{u} Y_{u}^{\dagger}\right)+\frac{17}{4} g_{1}^{2} \lambda_{4} \operatorname{Tr}\left(Y_{u} Y_{u}^{\dagger}\right)+\frac{45}{4} g_{2}^{2} \lambda_{4} \operatorname{Tr}\left(Y_{u} Y_{u}^{\dagger}\right)+40 g_{3}^{2} \lambda_{4} \operatorname{Tr}\left(Y_{u} Y_{u}^{\dagger}\right) \\
& -24 \lambda_{2} \lambda_{4} \operatorname{Tr}\left(Y_{u} Y_{u}^{\dagger}\right)-24 \lambda_{3} \lambda_{4} \operatorname{Tr}\left(Y_{u} Y_{u}^{\dagger}\right)-12 \lambda_{4}^{2} \operatorname{Tr}\left(Y_{u} Y_{u}^{\dagger}\right)-24 \lambda_{5}^{2} \operatorname{Tr}\left(Y_{u} Y_{u}^{\dagger}\right) \\
& -12 \lambda_{6} \lambda_{7} \operatorname{Tr}\left(Y_{u} Y_{u}^{\dagger}\right)-60 \lambda_{7}^{2} \operatorname{Tr}\left(Y_{u} Y_{u}^{\dagger}\right)-\frac{27}{2} \lambda_{4} \operatorname{Tr}\left(Y_{d} Y_{d}^{\dagger} Y_{d} Y_{d}^{\dagger}\right)+\frac{4}{5} g_{1}^{2} \operatorname{Tr}\left(Y_{d} Y_{u}^{\dagger} Y_{u} Y_{d}^{\dagger}\right) \\
& +64 g_{3}^{2} \operatorname{Tr}\left(Y_{d} Y_{u}^{\dagger} Y_{u} Y_{d}^{\dagger}\right)-24 \lambda_{3} \operatorname{Tr}\left(Y_{d} Y_{u}^{\dagger} Y_{u} Y_{d}^{\dagger}\right)-33 \lambda_{4} \operatorname{Tr}\left(Y_{d} Y_{u}^{\dagger} Y_{u} Y_{d}^{\dagger}\right) \\
& -\frac{9}{2} \lambda_{4} \operatorname{Tr}\left(Y_{e} Y_{e}^{\dagger} Y_{e} Y_{e}^{\dagger}\right)-\frac{27}{2} \lambda_{4} \operatorname{Tr}\left(Y_{u} Y_{u}^{\dagger} Y_{u} Y_{u}^{\dagger}\right)-12 \operatorname{Tr}\left(Y_{d} Y_{d}^{\dagger} Y_{d} Y_{u}^{\dagger} Y_{u} Y_{d}^{\dagger}\right) \\
& -12 \operatorname{Tr}\left(Y_{d} Y_{u}^{\dagger} Y_{u} Y_{d}^{\dagger} Y_{d} Y_{d}^{\dagger}\right)-24 \operatorname{Tr}\left(Y_{d} Y_{u}^{\dagger} Y_{u} Y_{u}^{\dagger} Y_{u} Y_{d}^{\dagger}\right) \text {, } \\
& \beta_{\lambda_{5}}^{(1)}=-\frac{9}{5} g_{1}^{2} \lambda_{5}-9 g_{2}^{2} \lambda_{5}+4 \lambda_{1} \lambda_{5}+4 \lambda_{2} \lambda_{5}+8 \lambda_{3} \lambda_{5}+12 \lambda_{4} \lambda_{5}+10 \lambda_{6}^{2}+4 \lambda_{6} \lambda_{7}+10 \lambda_{7}^{2} \\
& +6 \lambda_{5} \operatorname{Tr}\left(Y_{d} Y_{d}^{\dagger}\right)+2 \lambda_{5} \operatorname{Tr}\left(Y_{e} Y_{e}^{\dagger}\right)+6 \lambda_{5} \operatorname{Tr}\left(Y_{u} Y_{u}^{\dagger}\right) \text {, } \\
& \beta_{\lambda_{5}}^{(2)}=+\frac{1413}{200} g_{1}^{4} \lambda_{5}+\frac{57}{20} g_{1}^{2} g_{2}^{2} \lambda_{5}-\frac{231}{8} g_{2}^{4} \lambda_{5}-\frac{12}{5} g_{1}^{2} \lambda_{1} \lambda_{5}-28 \lambda_{1}^{2} \lambda_{5}-\frac{12}{5} g_{1}^{2} \lambda_{2} \lambda_{5}-28 \lambda_{2}^{2} \lambda_{5} \\
& +\frac{48}{5} g_{1}^{2} \lambda_{3} \lambda_{5}+36 g_{2}^{2} \lambda_{3} \lambda_{5}-80 \lambda_{1} \lambda_{3} \lambda_{5}-80 \lambda_{2} \lambda_{3} \lambda_{5}-28 \lambda_{3}^{2} \lambda_{5}+\frac{72}{5} g_{1}^{2} \lambda_{4} \lambda_{5}+72 g_{2}^{2} \lambda_{4} \lambda_{5} \\
& -88 \lambda_{1} \lambda_{4} \lambda_{5}-88 \lambda_{2} \lambda_{4} \lambda_{5}-76 \lambda_{3} \lambda_{4} \lambda_{5}-32 \lambda_{4}^{2} \lambda_{5}+6 \lambda_{5}^{3}+12 g_{1}^{2} \lambda_{6}^{2}+54 g_{2}^{2} \lambda_{6}^{2}-148 \lambda_{1} \lambda_{6}^{2} \\
& -20 \lambda_{2} \lambda_{6}^{2}-72 \lambda_{3} \lambda_{6}^{2}-76 \lambda_{4} \lambda_{6}^{2}-72 \lambda_{5} \lambda_{6}^{2}-\frac{12}{5} g_{1}^{2} \lambda_{6} \lambda_{7}-40 \lambda_{1} \lambda_{6} \lambda_{7}-40 \lambda_{2} \lambda_{6} \lambda_{7} \\
& -80 \lambda_{3} \lambda_{6} \lambda_{7}-88 \lambda_{4} \lambda_{6} \lambda_{7}-168 \lambda_{5} \lambda_{6} \lambda_{7}+12 g_{1}^{2} \lambda_{7}^{2}+54 g_{2}^{2} \lambda_{7}^{2}-20 \lambda_{1} \lambda_{7}^{2}-148 \lambda_{2} \lambda_{7}^{2} \\
& -72 \lambda_{3} \lambda_{7}^{2}-76 \lambda_{4} \lambda_{7}^{2}-72 \lambda_{5} \lambda_{7}^{2} \\
& +\frac{1}{4}\left(16\left(10 g_{3}^{2} \lambda_{5}-3\left(2 \lambda_{1} \lambda_{5}+2 \lambda_{3} \lambda_{5}+3 \lambda_{4} \lambda_{5}+5 \lambda_{6}^{2}+\lambda_{6} \lambda_{7}\right)\right)+45 g_{2}^{2} \lambda_{5}+5 g_{1}^{2} \lambda_{5}\right) \operatorname{Tr}\left(Y_{d} Y_{d}^{\dagger}\right) \\
& +\frac{1}{4}\left(15 g_{1}^{2} \lambda_{5}+15 g_{2}^{2} \lambda_{5}-16\left(2 \lambda_{1} \lambda_{5}+2 \lambda_{3} \lambda_{5}+3 \lambda_{4} \lambda_{5}+5 \lambda_{6}^{2}+\lambda_{6} \lambda_{7}\right)\right) \operatorname{Tr}\left(Y_{e} Y_{e}^{\dagger}\right) \\
& +\frac{17}{4} g_{1}^{2} \lambda_{5} \operatorname{Tr}\left(Y_{u} Y_{u}^{\dagger}\right)+\frac{45}{4} g_{2}^{2} \lambda_{5} \operatorname{Tr}\left(Y_{u} Y_{u}^{\dagger}\right)+40 g_{3}^{2} \lambda_{5} \operatorname{Tr}\left(Y_{u} Y_{u}^{\dagger}\right)-24 \lambda_{2} \lambda_{5} \operatorname{Tr}\left(Y_{u} Y_{u}^{\dagger}\right) \\
& -24 \lambda_{3} \lambda_{5} \operatorname{Tr}\left(Y_{u} Y_{u}^{\dagger}\right)-36 \lambda_{4} \lambda_{5} \operatorname{Tr}\left(Y_{u} Y_{u}^{\dagger}\right)-12 \lambda_{6} \lambda_{7} \operatorname{Tr}\left(Y_{u} Y_{u}^{\dagger}\right)-60 \lambda_{7}^{2} \operatorname{Tr}\left(Y_{u} Y_{u}^{\dagger}\right) \\
& -\frac{3}{2} \lambda_{5} \operatorname{Tr}\left(Y_{d} Y_{d}^{\dagger} Y_{d} Y_{d}^{\dagger}\right)-33 \lambda_{5} \operatorname{Tr}\left(Y_{d} Y_{u}^{\dagger} Y_{u} Y_{d}^{\dagger}\right)-\frac{1}{2} \lambda_{5} \operatorname{Tr}\left(Y_{e} Y_{e}^{\dagger} Y_{e} Y_{e}^{\dagger}\right)-\frac{3}{2} \lambda_{5} \operatorname{Tr}\left(Y_{u} Y_{u}^{\dagger} Y_{u} Y_{u}^{\dagger}\right),
\end{aligned}
$$




$$
\begin{aligned}
& \beta_{\lambda_{6}}^{(1)}=-\frac{9}{5} g_{1}^{2} \lambda_{6}-9 g_{2}^{2} \lambda_{6}+24 \lambda_{1} \lambda_{6}+6 \lambda_{3} \lambda_{6}+8 \lambda_{4} \lambda_{6}+10 \lambda_{5} \lambda_{6}+6 \lambda_{3} \lambda_{7}+4 \lambda_{4} \lambda_{7}+2 \lambda_{5} \lambda_{7} \\
& +9 \lambda_{6} \operatorname{Tr}\left(Y_{d} Y_{d}^{\dagger}\right)+3 \lambda_{6} \operatorname{Tr}\left(Y_{e} Y_{e}^{\dagger}\right)+3 \lambda_{6} \operatorname{Tr}\left(Y_{u} Y_{u}^{\dagger}\right) \text {, } \\
& \beta_{\lambda_{6}}^{(2)}=+\frac{1683}{200} g_{1}^{4} \lambda_{6}+\frac{87}{20} g_{1}^{2} g_{2}^{2} \lambda_{6}-\frac{141}{8} g_{2}^{4} \lambda_{6}+\frac{108}{5} g_{1}^{2} \lambda_{1} \lambda_{6}+108 g_{2}^{2} \lambda_{1} \lambda_{6}-318 \lambda_{1}^{2} \lambda_{6}+6 \lambda_{2}^{2} \lambda_{6} \\
& +\frac{18}{5} g_{1}^{2} \lambda_{3} \lambda_{6}+18 g_{2}^{2} \lambda_{3} \lambda_{6}-132 \lambda_{1} \lambda_{3} \lambda_{6}-36 \lambda_{2} \lambda_{3} \lambda_{6}-32 \lambda_{3}^{2} \lambda_{6}+6 g_{1}^{2} \lambda_{4} \lambda_{6}+36 g_{2}^{2} \lambda_{4} \lambda_{6} \\
& -140 \lambda_{1} \lambda_{4} \lambda_{6}-28 \lambda_{2} \lambda_{4} \lambda_{6}-68 \lambda_{3} \lambda_{4} \lambda_{6}-34 \lambda_{4}^{2} \lambda_{6}+12 g_{1}^{2} \lambda_{5} \lambda_{6}+54 g_{2}^{2} \lambda_{5} \lambda_{6}-148 \lambda_{1} \lambda_{5} \lambda_{6} \\
& -20 \lambda_{2} \lambda_{5} \lambda_{6}-72 \lambda_{3} \lambda_{5} \lambda_{6}-76 \lambda_{4} \lambda_{5} \lambda_{6}-36 \lambda_{5}^{2} \lambda_{6}-111 \lambda_{6}^{3}+\frac{27}{20} g_{1}^{4} \lambda_{7}+\frac{3}{2} g_{1}^{2} g_{2}^{2} \lambda_{7}+\frac{45}{4} g_{2}^{4} \lambda_{7} \\
& +\frac{36}{5} g_{1}^{2} \lambda_{3} \lambda_{7}+36 g_{2}^{2} \lambda_{3} \lambda_{7}-36 \lambda_{1} \lambda_{3} \lambda_{7}-36 \lambda_{2} \lambda_{3} \lambda_{7}-36 \lambda_{3}^{2} \lambda_{7}+\frac{24}{5} g_{1}^{2} \lambda_{4} \lambda_{7}+18 g_{2}^{2} \lambda_{4} \lambda_{7} \\
& -28 \lambda_{1} \lambda_{4} \lambda_{7}-28 \lambda_{2} \lambda_{4} \lambda_{7}-56 \lambda_{3} \lambda_{4} \lambda_{7}-34 \lambda_{4}^{2} \lambda_{7}-\frac{6}{5} g_{1}^{2} \lambda_{5} \lambda_{7}-20 \lambda_{1} \lambda_{5} \lambda_{7}-20 \lambda_{2} \lambda_{5} \lambda_{7} \\
& -40 \lambda_{3} \lambda_{5} \lambda_{7}-44 \lambda_{4} \lambda_{5} \lambda_{7}-42 \lambda_{5}^{2} \lambda_{7}-126 \lambda_{6}^{2} \lambda_{7}-33 \lambda_{6} \lambda_{7}^{2}-42 \lambda_{7}^{3} \\
& +\frac{3}{8}\left(16\left(10 g_{3}^{2}-24 \lambda_{1}-3 \lambda_{3}-4 \lambda_{4}-5 \lambda_{5}\right)+45 g_{2}^{2}+5 g_{1}^{2}\right) \lambda_{6} \operatorname{Tr}\left(Y_{d} Y_{d}^{\dagger}\right) \\
& +\frac{1}{8}\left(-16\left(24 \lambda_{1}+3 \lambda_{3}+4 \lambda_{4}+5 \lambda_{5}\right)+45 g_{1}^{2}+45 g_{2}^{2}\right) \lambda_{6} \operatorname{Tr}\left(Y_{e} Y_{e}^{\dagger}\right)+\frac{17}{8} g_{1}^{2} \lambda_{6} \operatorname{Tr}\left(Y_{u} Y_{u}^{\dagger}\right) \\
& +\frac{45}{8} g_{2}^{2} \lambda_{6} \operatorname{Tr}\left(Y_{u} Y_{u}^{\dagger}\right)+20 g_{3}^{2} \lambda_{6} \operatorname{Tr}\left(Y_{u} Y_{u}^{\dagger}\right)-18 \lambda_{3} \lambda_{6} \operatorname{Tr}\left(Y_{u} Y_{u}^{\dagger}\right)-24 \lambda_{4} \lambda_{6} \operatorname{Tr}\left(Y_{u} Y_{u}^{\dagger}\right) \\
& -30 \lambda_{5} \lambda_{6} \operatorname{Tr}\left(Y_{u} Y_{u}^{\dagger}\right)-36 \lambda_{3} \lambda_{7} \operatorname{Tr}\left(Y_{u} Y_{u}^{\dagger}\right)-24 \lambda_{4} \lambda_{7} \operatorname{Tr}\left(Y_{u} Y_{u}^{\dagger}\right)-12 \lambda_{5} \lambda_{7} \operatorname{Tr}\left(Y_{u} Y_{u}^{\dagger}\right) \\
& -\frac{33}{4} \lambda_{6} \operatorname{Tr}\left(Y_{d} Y_{d}^{\dagger} Y_{d} Y_{d}^{\dagger}\right)-21 \lambda_{6} \operatorname{Tr}\left(Y_{d} Y_{u}^{\dagger} Y_{u} Y_{d}^{\dagger}\right)-\frac{11}{4} \lambda_{6} \operatorname{Tr}\left(Y_{e} Y_{e}^{\dagger} Y_{e} Y_{e}^{\dagger}\right) \\
& -\frac{27}{4} \lambda_{6} \operatorname{Tr}\left(Y_{u} Y_{u}^{\dagger} Y_{u} Y_{u}^{\dagger}\right) \\
& \beta_{\lambda_{7}}^{(1)}=+6 \lambda_{3} \lambda_{6}+4 \lambda_{4} \lambda_{6}+2 \lambda_{5} \lambda_{6}-\frac{9}{5} g_{1}^{2} \lambda_{7}-9 g_{2}^{2} \lambda_{7}+24 \lambda_{2} \lambda_{7}+6 \lambda_{3} \lambda_{7}+8 \lambda_{4} \lambda_{7}+10 \lambda_{5} \lambda_{7} \\
& +3 \lambda_{7} \operatorname{Tr}\left(Y_{d} Y_{d}^{\dagger}\right)+\lambda_{7} \operatorname{Tr}\left(Y_{e} Y_{e}^{\dagger}\right)+9 \lambda_{7} \operatorname{Tr}\left(Y_{u} Y_{u}^{\dagger}\right) \\
& \beta_{\lambda_{7}}^{(2)}=+\frac{27}{20} g_{1}^{4} \lambda_{6}+\frac{3}{2} g_{1}^{2} g_{2}^{2} \lambda_{6}+\frac{45}{4} g_{2}^{4} \lambda_{6}+\frac{36}{5} g_{1}^{2} \lambda_{3} \lambda_{6}+36 g_{2}^{2} \lambda_{3} \lambda_{6}-36 \lambda_{1} \lambda_{3} \lambda_{6}-36 \lambda_{2} \lambda_{3} \lambda_{6} \\
& -36 \lambda_{3}^{2} \lambda_{6}+\frac{24}{5} g_{1}^{2} \lambda_{4} \lambda_{6}+18 g_{2}^{2} \lambda_{4} \lambda_{6}-28 \lambda_{1} \lambda_{4} \lambda_{6}-28 \lambda_{2} \lambda_{4} \lambda_{6}-56 \lambda_{3} \lambda_{4} \lambda_{6}-34 \lambda_{4}^{2} \lambda_{6} \\
& -\frac{6}{5} g_{1}^{2} \lambda_{5} \lambda_{6}-20 \lambda_{1} \lambda_{5} \lambda_{6}-20 \lambda_{2} \lambda_{5} \lambda_{6}-40 \lambda_{3} \lambda_{5} \lambda_{6}-44 \lambda_{4} \lambda_{5} \lambda_{6}-42 \lambda_{5}^{2} \lambda_{6}-42 \lambda_{6}^{3}+\frac{1683}{200} g_{1}^{4} \lambda_{7} \\
& +\frac{87}{20} g_{1}^{2} g_{2}^{2} \lambda_{7}-\frac{141}{8} g_{2}^{4} \lambda_{7}+6 \lambda_{1}^{2} \lambda_{7}+\frac{108}{5} g_{1}^{2} \lambda_{2} \lambda_{7}+108 g_{2}^{2} \lambda_{2} \lambda_{7}-318 \lambda_{2}^{2} \lambda_{7}+\frac{18}{5} g_{1}^{2} \lambda_{3} \lambda_{7} \\
& +18 g_{2}^{2} \lambda_{3} \lambda_{7}-36 \lambda_{1} \lambda_{3} \lambda_{7}-132 \lambda_{2} \lambda_{3} \lambda_{7}-32 \lambda_{3}^{2} \lambda_{7}+6 g_{1}^{2} \lambda_{4} \lambda_{7}+36 g_{2}^{2} \lambda_{4} \lambda_{7}-28 \lambda_{1} \lambda_{4} \lambda_{7} \\
& -140 \lambda_{2} \lambda_{4} \lambda_{7}-68 \lambda_{3} \lambda_{4} \lambda_{7}-34 \lambda_{4}^{2} \lambda_{7}+12 g_{1}^{2} \lambda_{5} \lambda_{7}+54 g_{2}^{2} \lambda_{5} \lambda_{7}-20 \lambda_{1} \lambda_{5} \lambda_{7}-148 \lambda_{2} \lambda_{5} \lambda_{7} \\
& -72 \lambda_{3} \lambda_{5} \lambda_{7}-76 \lambda_{4} \lambda_{5} \lambda_{7}-36 \lambda_{5}^{2} \lambda_{7}-33 \lambda_{6}^{2} \lambda_{7}-126 \lambda_{6} \lambda_{7}^{2}-111 \lambda_{7}^{3}-\frac{1}{8}\left(144 \lambda_{3}\left(2 \lambda_{6}+\lambda_{7}\right)\right. \\
& \left.-160 g_{3}^{2} \lambda_{7}+192 \lambda_{4}\left(\lambda_{6}+\lambda_{7}\right)+240 \lambda_{5} \lambda_{7}-45 g_{2}^{2} \lambda_{7}-5 g_{1}^{2} \lambda_{7}+96 \lambda_{5} \lambda_{6}\right) \operatorname{Tr}\left(Y_{d} Y_{d}^{\dagger}\right)
\end{aligned}
$$




$$
\begin{aligned}
& -\frac{1}{8}\left(-15 g_{1}^{2} \lambda_{7}-15 g_{2}^{2} \lambda_{7}+32 \lambda_{5} \lambda_{6}+48 \lambda_{3}\left(2 \lambda_{6}+\lambda_{7}\right)+64 \lambda_{4}\left(\lambda_{6}+\lambda_{7}\right)+80 \lambda_{5} \lambda_{7}\right) \operatorname{Tr}\left(Y_{e} Y_{e}^{\dagger}\right) \\
& +\frac{51}{8} g_{1}^{2} \lambda_{7} \operatorname{Tr}\left(Y_{u} Y_{u}^{\dagger}\right)+\frac{135}{8} g_{2}^{2} \lambda_{7} \operatorname{Tr}\left(Y_{u} Y_{u}^{\dagger}\right)+60 g_{3}^{2} \lambda_{7} \operatorname{Tr}\left(Y_{u} Y_{u}^{\dagger}\right)-144 \lambda_{2} \lambda_{7} \operatorname{Tr}\left(Y_{u} Y_{u}^{\dagger}\right) \\
& -18 \lambda_{3} \lambda_{7} \operatorname{Tr}\left(Y_{u} Y_{u}^{\dagger}\right)-24 \lambda_{4} \lambda_{7} \operatorname{Tr}\left(Y_{u} Y_{u}^{\dagger}\right)-30 \lambda_{5} \lambda_{7} \operatorname{Tr}\left(Y_{u} Y_{u}^{\dagger}\right)-\frac{27}{4} \lambda_{7} \operatorname{Tr}\left(Y_{d} Y_{d}^{\dagger} Y_{d} Y_{d}^{\dagger}\right) \\
& -21 \lambda_{7} \operatorname{Tr}\left(Y_{d} Y_{u}^{\dagger} Y_{u} Y_{d}^{\dagger}\right)-\frac{9}{4} \lambda_{7} \operatorname{Tr}\left(Y_{e} Y_{e}^{\dagger} Y_{e} Y_{e}^{\dagger}\right)-\frac{33}{4} \lambda_{7} \operatorname{Tr}\left(Y_{u} Y_{u}^{\dagger} Y_{u} Y_{u}^{\dagger}\right) .
\end{aligned}
$$

[1] G. Aad et al. (ATLAS Collaboration), Observation of a new particle in the search for the Standard Model Higgs boson with the ATLAS detector at the LHC, Phys. Lett. B 716, 1 (2012).

[2] S. Chatrchyan et al. (CMS Collaboration), Observation of a new boson at a mass of $125 \mathrm{GeV}$ with the CMS experiment at the LHC, Phys. Lett. B 716, 30 (2012).

[3] J. McDowall and D. J. Miller, High scale boundary conditions with an additional complex singlet, Phys. Rev. D 97, 115042 (2018).

[4] G. Degrassi, S. Di Vita, J. Elias-Miro, J. R. Espinosa, G. F. Giudice, G. Isidori, and A. Strumia, Higgs mass and vacuum stability in the Standard Model at NNLO, J. High Energy Phys. 08 (2012) 098.

[5] M. Holthausen, K. S. Lim, and M. Lindner, Planck scale boundary conditions and the Higgs mass, J. High Energy Phys. 02 (2012) 037.

[6] G. Iacobellis and I. Masina, Stationary configurations of the Standard Model Higgs potential: Electroweak stability and rising inflection point, Phys. Rev. D 94, 073005 (2016).

[7] N. Haba, K. Kaneta, and R. Takahashi, Planck scale boundary conditions in the standard model with singlet scalar dark matter, J. High Energy Phys. 04 (2014) 029.

[8] A. Eichhorn and M. M. Scherer, Planck scale, Higgs mass, and scalar dark matter, Phys. Rev. D 90, 025023 (2014).

[9] N. Khan and S. Rakshit, Study of electroweak vacuum metastability with a singlet scalar dark matter, Phys. Rev. D 90, 113008 (2014).

[10] N. Khan and S. Rakshit, Constraints on inert dark matter from the metastability of the electroweak vacuum, Phys. Rev. D 92, 055006 (2015).

[11] A. J. Helmboldt, P. Humbert, M. Lindner, and J. Smirnov, Minimal conformal extensions of the Higgs sector, J. High Energy Phys. 07 (2017) 113.

[12] I. Garg, S. Goswami, K. N. Vishnudath, and N. Khan, Electroweak vacuum stability in presence of singlet scalar dark matter in tev scale seesaw models, Phys. Rev. D 96, 055020 (2017).

[13] N. Khan, Exploring the hyperchargeless Higgs triplet model up to the Planck scale, Eur. Phys. J. C 78, 341 (2018).

[14] P. S. Bhupal Dev and A. Pilaftsis, Maximally symmetric two Higgs doublet model with natural Standard Model alignment, J. High Energy Phys. 12 (2014) 024; Erratum, J. High Energy Phys. 11 (2015) 147(E).

[15] P. S. Bhupal Dev and A. Pilaftsis, Natural alignment in the two Higgs doublet model, J. Phys. Conf. Ser. 873, 012008 (2017).

[16] G. Aad et al. (ATLAS and CMS Collaboration), Combined Measurement of the Higgs Boson Mass in $p p$ Collisions at $\sqrt{s}=7$ and $8 \mathrm{TeV}$ with the ATLAS and CMS Experiments, Phys. Rev. Lett. 114, 191803 (2015).

[17] D. Buttazzo, G. Degrassi, P. P. Giardino, G. F. Giudice, F. Sala, A. Salvio, and A. Strumia, Investigating the nearcriticality of the Higgs boson, J. High Energy Phys. 12 (2013) 089.

[18] N. Craig, The state of supersymmetry after run I of the LHC, arXiv:1309.0528.

[19] V. Khachatryan et al. (CMS Collaboration), Constraints on the spin-parity and anomalous HVV couplings of the Higgs boson in proton collisions at 7 and $8 \mathrm{TeV}$, Phys. Rev. D 92, 012004 (2015).

[20] V. Khachatryan et al. (CMS Collaboration), Precise determination of the mass of the Higgs boson and tests of compatibility of its couplings with the standard model predictions using proton collisions at 7 and $8 \mathrm{TeV}$, Eur. Phys. J. C 75, 212 (2015).

[21] G. Aad et al. (ATLAS Collaboration), Measurements of the Higgs boson production and decay rates and coupling strengths using pp collision data at $\sqrt{s}=7$ and $8 \mathrm{TeV}$ in the ATLAS experiment, Eur. Phys. J. C 76, 6 (2016).

[22] G. C. Branco, P. M. Ferreira, L. Lavoura, M. N. Rebelo, M. Sher, and J. P. Silva, Theory and phenomenology of twoHiggs-doublet models, Phys. Rep. 516, 1 (2012).

[23] A. Ilnicka, M. Krawczyk, and T. Robens, Inert doublet model in light of LHC run I and astrophysical data, Phys. Rev. D 93, 055026 (2016).

[24] I. P. Ivanov, Minkowski space structure of the Higgs potential in 2HDM, Phys. Rev. D 75, 035001 (2007); Erratum, Phys. Rev. D 76, 039902(E) (2007).

[25] P. Ferreira, H. E. Haber, and E. Santos, Preserving the validity of the Two-Higgs doublet model up to the Planck scale, Phys. Rev. D 92, 033003 (2015).

[26] F. Staub, SARAH 4: A tool for (not only SUSY) model builders, Comput. Phys. Commun. 185, 1773 (2014). 
[27] P. Athron, J.-h. Park, D. Stockinger, and A. Voigt, FlexibleSUSY-A spectrum generator generator for supersymmetric models, Comput. Phys. Commun. 190, 139 (2015).

[28] P. Athron, M. Bach, D. Harries, T. Kwasnitza, J.-h. Park, D. Stöckinger, A. Voigt, and J. Ziebell, FlexibleSUSY 2.0: Extensions to investigate the phenomenology of SUSY and non-SUSY models, Comput. Phys. Commun. 230, 145 (2018).

[29] B. C. Allanach, SOFTSUSY: A program for calculating supersymmetric spectra, Comput. Phys. Commun. 143, 305 (2002).

[30] B. C. Allanach, P. Athron, L. C. Tunstall, A. Voigt, and A. G. Williams, Next-to-minimal SOFTSUSY, Comput. Phys. Commun. 185, 2322 (2014).

[31] A. Datta, N. Ganguly, N. Khan, and S. Rakshit, Exploring collider signatures of the inert higgs doublet model, Phys. Rev. D 95, 015017 (2017).

[32] N. Khan and S. Rakshit, Constraints on inert dark matter from the metastability of the electroweak vacuum, Phys. Rev. D 92, 055006 (2015).

[33] A. Goudelis, B. Herrmann, and O. Stål, Dark matter in the inert doublet model after the discovery of a Higgs-like boson at the LHC, J. High Energy Phys. 09 (2013) 106.

[34] D. Chowdhury and O. Eberhardt, Global fits of the two-loop renormalized two-Higgs-doublet model with soft $\mathrm{Z}_{2}$ breaking, J. High Energy Phys. 11 (2015) 052.

[35] M. Sher, Electroweak Higgs potentials and vacuum stability, Phys. Rep. 179, 273 (1989).

[36] L. Chataignier, T. Prokopec, M. G. Schmidt, and B. Swiezewska, Single-scale renormalisation group improvement of multi-scale effective potentials, J. High Energy Phys. 03 (2018) 014.

[37] J. E. Camargo-Molina, B. O'Leary, W. Porod, and F. Staub, Vevacious: A tool for finding the global minima of one-loop effective potentials with many scalars, Eur. Phys. J. C 73, 2588 (2013).

[38] D. Eriksson, J. Rathsman, and O. Stal, 2HDMC: TwoHiggs-doublet model calculator physics and manual, Comput. Phys. Commun. 181, 189 (2010).

[39] P. Bechtle, O. Brein, S. Heinemeyer, O. Stal, T. Stefaniak, G. Weiglein, and K. E. Williams, HiggsBounds-4: Improved tests of extended Higgs sectors against exclusion bounds from LEP, the Tevatron and the LHC, Eur. Phys. J. C 74, 2693 (2014).

[40] P. Bechtle, S. Heinemeyer, O. Stal, T. Stefaniak, and G. Weiglein, HiggsSignals: Confronting arbitrary Higgs sectors with measurements at the Tevatron and the LHC, Eur. Phys. J. C 74, 2711 (2014).

[41] M. Gustafsson, E. Lundstrom, L. Bergstrom, and J. Edsjo, Significant Gamma Lines from Inert Higgs Dark Matter, Phys. Rev. Lett. 99, 041301 (2007).

[42] Q.-H. Cao, E. Ma, and G. Rajasekaran, Observing the dark scalar doublet and its impact on the Standard-Model Higgs boson at colliders, Phys. Rev. D 76, 095011 (2007).

[43] E. Lundstrom, M. Gustafsson, and J. Edsjo, The inert doublet model and LEP II limits, Phys. Rev. D 79, 035013 (2009).
[44] A. Pierce and J. Thaler, Natural dark matter from an unnatural Higgs boson and new colored particles at the TeV scale, J. High Energy Phys. 08 (2007) 026.

[45] C. Patrignani et al. (Particle Data Group), Review of particle physics, Chin. Phys. C 40, 100001 (2016).

[46] F. Mahmoudi, SuperIso: A program for calculating the isospin asymmetry of $B \rightarrow K^{*}$ gamma in the MSSM, Comput. Phys. Commun. 178, 745 (2008).

[47] F. Mahmoudi, SuperIso v2.3: A program for calculating flavor physics observables in supersymmetry, Comput. Phys. Commun. 180, 1579 (2009).

[48] F. Mahmoudi, SuperIso v3.0, flavor physics observables calculations: Extension to NMSSM, Comput. Phys. Commun. 180, 1718 (2009).

[49] G. Bélanger, F. Boudjema, A. Pukhov, and A. Semenov, micrOMEGAs4.1: Two dark matter candidates, Comput. Phys. Commun. 192, 322 (2015).

[50] G. Hinshaw et al. (WMAP Collaboration), Nine-year Wilkinson microwave anisotropy probe (WMAP) observations: Cosmological parameter results, Astrophys. J. Suppl. Ser. 208, 19 (2013).

[51] P. A. R. Ade et al. (Planck Collaboration), Planck 2015 results. XIII. Cosmological parameters, Astron. Astrophys. 594, A13 (2016).

[52] D. S. Akerib et al. (LUX Collaboration), Results from a Search for Dark Matter in the Complete LUX Exposure, Phys. Rev. Lett. 118, 021303 (2017).

[53] E. Aprile et al. (XENON Collaboration), Dark Matter Search Results from a One Tonne $\times$ Year Exposure of XENON1T, Phys. Rev. Lett. 121, 111302 (2018).

[54] G. Bhattacharyya, D. Das, M. J. Pérez, I. Saha, A. Santamaria, and O. Vives, Can measurements of 2HDM parameters provide hints for high scale supersymmetry?, Phys. Rev. D 97, 095018 (2018).

[55] C. D. Froggatt and H. B. Nielsen, Standard Model criticality prediction: Top mass $173 \pm 5 \mathrm{GeV}$ and Higgs mass $135 \pm 9$ GeV, Phys. Lett. B 368, 96 (1996).

[56] K. Kawana, Multiple point principle of the Standard Model with scalar singlet dark matter and right handed neutrinos, Prog. Theor. Exp. Phys. 2015, 023 B04 (2015).

[57] N. Haba, H. Ishida, N. Okada, and Y. Yamaguchi, Multiplepoint principle with a scalar singlet extension of the Standard Model, Prog. Theor. Exp. Phys. 2017, 013B03 (2017).

[58] Y. Hamada, H. Kawai, and K.-y. Oda, Predictions on mass of Higgs portal scalar dark matter from Higgs inflation and flat potential, J. High Energy Phys. 07 (2014) 026.

[59] H. F. B. Nielsen, D. L. Bennett, C. R. Das, C. D. Froggatt, and L. V. Laperashvili, F(750), we miss you as a bound state of 6 top and 6 antitop quarks, multiple point principle, Proc. Sci. CORFU2016 (2017) 050.

[60] C. D. Froggatt, L. V. Laperashvili, R. B. Nevzorov, H. B. Nielsen, and M. Sher, The two Higgs doublet model and the multiple point principle, arXiv:hep-ph/0412333.

[61] C. D. Froggatt, L. Laperashvili, R. Nevzorov, H. B. Nielsen, and M. Sher, Implementation of the multiple point principle in the two-Higgs doublet model of type II, Phys. Rev. D 73, 095005 (2006). 
[62] C. D. Froggatt, R. Nevzorov, H. B. Nielsen, and D. Thompson, Fixed point scenario in the two Higgs doublet Model inspired by degenerate vacua, Phys. Lett. B 657, 95 (2007).

[63] D. F. Litim and F. Sannino, Asymptotic safety guaranteed, J. High Energy Phys. 12 (2014) 178.

[64] D. F. Litim, M. Mojaza, and F. Sannino, Vacuum stability of asymptotically safe gauge-Yukawa theories, J. High Energy Phys. 01 (2016) 081.

[65] A. D. Bond, G. Hiller, K. Kowalska, and D. F. Litim, Directions for model building from asymptotic safety, J. High Energy Phys. 08 (2017) 004.

[66] A. D. Bond and D. F. Litim, Theorems for asymptotic safety of gauge theories, Eur. Phys. J. C 77, 429 (2017).

[67] F. Sannino and I. M. Shoemaker, Asymptotically safe dark matter, Phys. Rev. D 92, 043518 (2015).

[68] B. Bajc and F. Sannino, Asymptotically safe grand unification, J. High Energy Phys. 12 (2016) 141.

[69] G. M. Pelaggi, F. Sannino, A. Strumia, and E. Vigiani, Naturalness of asymptotically safe Higgs, Front. Phys. 5, 49 (2017).
[70] G. M. Pelaggi, A. D. Plascencia, A. Salvio, F. Sannino, J. Smirnov, and A. Strumia, Asymptotically safe Standard Model extensions, Phys. Rev. D 97, 095013 (2018).

[71] S. Ipek, A. D. Plascencia, and J. Turner, Assessing perturbativity and vacuum stability in high-scale leptogenesis, J. High Energy Phys. 12 (2018) 111.

[72] M. Shaposhnikov and C. Wetterich, Asymptotic safety of gravity and the Higgs boson mass, Phys. Lett. B 683, 196 (2010).

[73] C. Wetterich, Where to look for solving the gauge hierarchy problem?, Phys. Lett. B 718, 573 (2012).

[74] A. Eichhorn, S. Lippoldt, and V. Skrinjar, Nonminimal hints for asymptotic safety, Phys. Rev. D 97, 026002 (2018).

[75] A. Eichhorn and F. Versteegen, Upper bound on the Abelian gauge coupling from asymptotic safety, J. High Energy Phys. 01 (2018) 030.

[76] A. Eichhorn and A. Held, Top mass from asymptotic safety, Phys. Lett. B 777, 217 (2018). 